\title{
Partial identification of willingness-to-pay using shape restrictions with an application to the value of a statistical life
}

\author{
Aaron Sojourner*
}

January 12, 2010

\begin{abstract}
Economists often analyze cross-sectional data to estimate the value people implicit place on attributes of goods using hedonic methods. Usually strong enough assumptions are made on the functional form of utility to point identify individuals' willingness-to-pay (WTP) for changes in attribute levels. Instead, this paper develops a new way to partially identify WTP under a weak set of conditions on the shape of individual indifference curves. In particular, indifference curves are assumed to be increasing and convex in an attribute-cost space that is finitely bounded above. These shape restrictions provide informative partial identification without relying on functional form restrictions for utility. Identification given general, potentially discrete, as well as smooth price functions is analyzed. To illustrate this method, we contribute to the literature on the value of a statistical life (VSL) by analyzing labor market data to study people's willingness to pay (WTP) for reductions in levels of fatal risk. The paper contrasts VSL estimates from conventional analysis with the bounds obtained under this new approach using a common data set. The data are shown to be consistent with a wide range of WTP values even given equilibrium and credible shape restrictions. This suggests that conventional estimates may be driven by functional form restrictions imposed on utility rather than by the data or properties of equilibrium.
\end{abstract}

JEL Classifications: C14, I10, J28

*University of Minnesota, Carlson School of Management, Department of Human Resources and Industrial Relations. asojourn@umn.edu. Thanks to Pat Bajari, Charles Manski, Rosa Matzkin, James Roberts and Chris Taber for their guidance and assistance. Thanks to John Leeth and John Ruser for making their data available. All errors are mine. 


\section{Introduction}

Economists often use hedonic analysis to learn about people's preferences for particular attributes of goods when explicit prices for each attribute separately are not available. Attribute prices are not available because bundles of attributes, embodied in goods, are traded in markets but the attributes themselves are not. Studied attributes of goods include fatal occupational risks of jobs, air quality near houses [reviewed in Smith and Huang (1995)], processor speed in computers [Pakes (2003)] and many others. Contingentvaluation surveys and research designs based on policy discontinuities [e.g, Ashenfelter and Greenstone (2004) and Powell (2009) on fatal risk; Black (1999) on school quality; Chay and Greenstone (2005) on air quality] provide alternatives to hedonic analysis.

This paper focuses on identification of marginal willingness-to-pay (WTP) in a hedonic model. How much would we need to compensate people to keep them indifferent between some status quo and a ceteris paribus world where their consumption of the hedonic good is changed marginally along one dimension? Hedonic analysis has been used to study individual WTP and population average WTP (AWTP) for marginal changes in many attributes of jobs, houses, and consumer products. This paper develops and applies its results in a hedonic analysis of labor market choice, where workers trade wages against fatal occupational risk in choosing a job. However, the identification approach developed here could apply in any of these other hedonic settings.

There are two central challenges in hedonic analysis; this paper innovates on the second challenge. The first challenge is to identify the set of choices available to each individual when only their realized choices are observed. Knowing what options each individual passed up in making the observed choice is the foundation for insight into preferences. A choice set is commonly characterized as a function mapping hedonic attributes into prices for a given individual consumer or worker. Although credibly identifying this choice set is not trivial, this paper assumes it has been met in order to focus attention on the innovation made towards the second challenge. That said, this paper does study identification assuming price functions with a range of properties. This is important because recent work [Eckland, Heckman, and Nesheim (2004); Bajari and Benkard (2005); Pakes (2008)] has highlighted the relevance of considering discontinuous and potentially discrete market price curves in hedonics. The second challenge is to translate information about each consumer's revealed preference for his or her observed choice into a useful characterization of preferences. The paper's main contribution lies here.

This paper develops a new approach to identification of willingness-to-pay (WTP) in hedonic models based on imposing shape restrictions on individuals' indifference curves. The shape restrictions derive from weak conditions on preferences and generate informative partial identification of WTP given cross-sectional data from a single market. The critical conditions are that individual's indifference curves are assumed to be increasing and convex in a finitely-bounded risk $\times$ wage (attribute $\times$ price) space. These shape restrictions partially-identify individual WTP for changes in risk along the status-quo indifference curve, i.e. they imply bounds on the indifference curve. ${ }^{1}$ These bounds depend on the econometrician's choice of parameters for the upper bounds on the attribute $\times$ price space,

\footnotetext{
${ }^{1}$ This builds from analysis of shape-restricted treatment response in Manski (2003).
} 
as explored in the model and the application. Combining the shape restrictions with information about the price function produces tighter bounds on WTP and AWTP. These bounds are described by a series of propositions throughout Section 2.

This shape-restriction approach adds a new kind of tool to the hedonics literature, which has generally relied on functional form assumptions on utility to point-identify (or over-identify) preferences. One common approach is to assume that individuals have homogeneous preferences. This permits interpretation of the price function itself as an indifference curve and of the function's slope as AWTP. Although the theoretical limitations of this approach are clear, many empirical papers estimate AWTP this way. Allowing for heterogeneous preferences, the most common approach is to assume a convenient functional form for utility that enables point identification of preferences from observation of a single choice. These are typically either parametric or rely on additive separability of the preference heterogeneity parameter [Eckland, Heckman, and Nesheim (2004)]. Recently, Heckman, Matzkin, and Nesheim (2009) proposed a strictly more general approach to point-identify nonparametric, nonadditive preferences assuming that, in the equation describing individuals' marginal utility, preference heterogeneity is separable from observed characteristics of individuals and hedonic goods. In weakening structural requirements to obtain point-identification, this is a large theoretical step forward. However, in each case, the right kind of functional form on utility is imposed so that preferences can be point-identified.

In contrast, the bounds obtained in this paper describe the set of WTP values that would be generated by considering the union of all utility functions (or underlying preferences) consistent with the assumed shape restrictions on indifference curves. Observing an individual making a single choice cannot give solid ground for a rich characterization of preferences. If one demands point identification of preferences, one must necessarily invoke strong structure. This is a fundamental problem in hedonic analysis of cross-sectional data. Rather than pushing so hard for AN answer, this paper instead asks what range of answers are consistent with the data and credible economic restrictions on preferences.

A few other papers have studied partial identification in hedonic models. Motivated by the new-goods problem in construction of price indices and building on early work by Konüs (1939), Pakes (2003) develops bounds on the compensating variation necessary to hold welfare constant as prices and choice sets change over time. He does not study the additional identifying power of shape-restricted preferences. Bajari and Benkard (2005) study partial identification of parametric consumer preferences in differentiated product markets characterized by discrete price functions in order to identify demand and welfare. Their parametric assumptions are strong enough to deliver point identification of preferences from cross-sectional choice data assuming a smooth price function.

This paper develops and applies its model in the context of one active, important strand of the hedonics literature - studying cross-sectional labor market data to estimate AWTP for a marginal change in fatal risk. Since the seminal work of Rosen and Thaler (1976), a large literature has used this approach to estimate the value of a statistical life (VSL). Appealing to the theory of compensating differentials, workers in labor market data are assumed to have implicitly traded wages against occupational safety in choosing their preferred job. Understanding the value people implicitly put on risk is useful in 
policymaking. In cost-benefit analysis, some factors are easy to monetize because they commonly trade in the marketplace and have well-established prices, such as the cost of installing a smokestack scrubber. However, some factors are difficult to monetize, even if their non-monetary benefits are understood. In order to summarize the trade-off between money and risk, economists use the concept of VSL.

A simple example illustrates the reasoning. Suppose:

1. $N$ people are exposed to a fatal risk at level $r=\operatorname{Pr}($ death).

2. Individual- $n$ is willing to pay $W T P_{n}$ to move to risk level $r-\Delta$, meaning individual- $n$ is indifferent between the money-risk bundles $(0, r)$ and $\left(-W T P_{n}, r-\Delta\right)$.

3. Implementing policy would change the risk level from $r$ to $r-\Delta$ and $\operatorname{cost} C$.

Simple cost-benefit analysis suggests adopting policy if and only if $C \leq \sum W T P_{n}$. To facilitate generalization across samples, levels of risk $r$, and risk increments $\Delta$, the value of risk reduction is summarized as the value of a statistical life: $V S L(r, \Delta) \equiv$ $(N \Delta)^{-1} \sum W T P_{n}$. This is the average willingness to pay (AWTP) for a change in risk from some given level $r$ to the new level $r-\Delta$, scaled by the increment of change. In the literature, the VSL estimates most often presented are estimates of the AWTP for a marginal change $(\Delta \rightarrow 0)$ in risk from the sample mean or median level of risk. This is equivalent to the average slope of individual risk-wage indifference curves at that level of risk across the population.

Economists' VSL estimates impact policies and lives. Many federal agencies use these estimates to guide decision-making. Under-estimates of VSL mean that not enough lives are saved. Over-estimates mean that resources are wasted. U.S. Environmental Protection Agency guidelines recommend use of a VSL of $\$ 6.2$ million (2000 US\$). They chose this value by calculating the arithmetic mean of 26 studies reviewed in Viscusi (1992). In a February 5, 2009 memo, the Department of Transportation raised its baseline VSL for costbenefit analyses to $\$ 5.8$ million, "based on our improved understanding of the academic research literature [Duval and Gribbin (2009)]." The new baseline VSL is set equal to the mean of 5 VSL estimates from recent analyses and meta-analyses [Mrozek and Taylor (2002); Miller (2000); Viscusi (2004); Kochi, Hubbell, and Kramer (2006); Viscusi and Aldy (2003)].

Unfortunately, estimates of VSL in the literature vary quite widely. Conventional models assuming homogeneous preferences or functional forms for utility applied to U.S. labor market data generally produce VSL estimates in the range of $\$ 4$-9 million although the literature contains much higher and lower estimates [Viscusi and Aldy (2003)]. Other researchers have used alternative strategies to estimate VSL and have also obtained widely varying estimates. For instance, Ashenfelter and Greenstone (2004) use changes in state speed limits to estimate AWTP for risk reductions and get an imprecise point estimate of \$1.54 million for VSL. Powell (2009) exploits variation in marginal tax rates across time and and gets a VSL estimate an between $\$ 50$ and $\$ 75$ million. To reflect uncertainty about VSL, the U.S. Department of Transportation now requires that cost-benefit analyses be 
presented based on VSL of $\$ 3.2$ and $\$ 8.4$ million as well. These widely varying estimates trouble researchers and policy makers, who would prefer a converging set of evidence. ${ }^{2}$

The later sections of the paper apply this model to the kind of labor market data conventionally used in VSL analysis and estimates bounds on VSL. Section 3.1 gives detail about the data, which were previously used in Leeth and Ruser (2003a) and generously shared. Section 4 presents results. This new approach illuminates the degree to which point estimates in the VSL literature are due to data and to what degree they are due to strong, convenient conditions imposed on utility. Observed data are shown to be consistent with quite high and low VSL estimates. This wide range of possibility may help explain why VSL estimates vary across studies with different specifications and models. The tightest bounds estimated here are wide, between $\$ 2.3$ and $\$ 58$ million dollars, although they do exclude some of the highest and lowest point estimates in the literature. Assuming different values for the bounds on the risk $\times$ wage space gives wider bounds. Section 5 concludes. An appendix provides formal proof of the propositions as well as intermediate results. Additional tables and figures follow.

\section{Model}

\subsection{Model with shape restrictions alone}

A simple and weak nonparametric version of the model is presented to clarify the main ideas. The goal is to illuminate the identifying power of shape restrictions on indifference curves as well as the connection between bounds on indifference curves, on WTP, and on AWTP. The objective is not to generate strong results or to exploit rich detail. Later sections move in that direction by adding more structure to the model of the market.

Jobs differ in their level of fatal occupational risk, $r \in R \subseteq \mathbb{R}$. This affects worker utility and firm profits and, hence, influence wages, $w \in W \subseteq \mathbb{R}$. Jobs are characterized by their risk level and wage, that is their hedonic attribute and price. The econometrician observes a random sample of $N$ jobs, $\left\{\left(r_{n}, w_{n}\right)\right\}_{n=1}^{N}$ from the population.

Each individual worker's preferences over jobs can be expressed as either an individualspecific utility function given any job, $U_{n}(r, w)$, or a set of indifference curves in $R \times W$. Throughout the paper, attention will focus on one indifference curve for each individual the curve passing through $\left(r_{n}, w_{n}\right)$, the worker's observed, status quo job. This particular curve is conventionally the focus of WTP estimation in the VSL literature and elsewhere. It characterizes how much would someone be willing to pay to move from the status quo to an alternative that differs only in its level of the hedonic attribute. The curve's slope describes WTP for a marginal change in attribute level from a given base level.

\footnotetext{
2 "Research into these values has been pursued for a generation, and estimating techniques, model specifications, and sources of data have continued to evolve. Nevertheless, the uncertainty of estimates has not been substantially reduced.... The standard we are adopting may be seen as a central tendency, but there can be no assurance that the assumption of higher or lower values would not improve the net benefits of decisions. Therefore, examination of a range of alternative values must be regarded as an essential component of the analytical process [Duval and Gribbin (2009)]."
} 
Consider any worker- $n$ observed in job $\left(r_{n}, w_{n}\right)$. Her status-quo utility level is $\bar{u}_{n} \equiv$ $U_{n}\left(r_{n}, w_{n}\right)$. The indifference curve in attribute-price space $R \times W$ passing through this job contains all job types that also produce utility $\bar{u}_{n}$ for individual- $n$. Let the function $\theta_{n}: R \rightarrow W$ be implicitly defined as equivalent to this status-quo indifference curve; $\theta_{n}$ is defined such that $\bar{u}_{n}=U_{n}\left(r, \theta_{n}(r)\right)$. Given a risk level of interest, $r, \theta_{n}(\cdot)$ returns the wage required to keep individual- $n$ indifferent between her status-quo job and a job with risk level $r$. It is immediate that $w_{n}=\theta_{n}\left(r_{n}\right)$.

To understand VSL, we study individuals' WTP for marginal changes in risk from a given base level of risk $r$, that is the slope of the indifference curve, $\theta^{\prime}(r)$. Usually, interest focuses on the sample mean or median level of risk. From the implicit function theorem,

$$
\theta_{n}^{\prime}(r)=-\left.\frac{\frac{\partial U_{n}(r, w)}{\partial r}}{\frac{\partial U_{n}(r, w)}{\partial w}}\right|_{\left(r, w=\theta_{n}(r)\right)}
$$

This $\theta_{n}^{\prime}(r)$ is the marginal rate of substitution of risk for wages at $r$. Since we focus on partial identification, we will ask what range of values of $\theta_{n}^{\prime}$ are consistent with data and the model. Following the VSL literature, we will aggregate across individuals to study AWTP, denoted $E \theta^{\prime}$ with the expectation taken across the population distribution.

Individual indifference curves are assumed to have the following properties.

Condition 1. $\theta_{n}$ is

a Weakly increasing: $\theta_{n}(r) \leq \theta_{n}(s)$ for $r<s$

b Weakly convex: $\frac{\theta_{n}(s)-\theta_{n}(r)}{s-r} \leq \frac{\theta_{n}(t)-\theta_{n}(s)}{t-s}$ for $r<s<t$

For notational convenience, $\theta_{n}$ is assumed twice differentiable. ${ }^{3}$ Then, C1.a is $\theta_{n}^{\prime} \geq 0$ and C1.b is $\theta_{n}^{\prime \prime} \geq 0$. The substantive results hold without differentiability.

C1.a is uncontroversial. It requires only that a worker offered an alternative job at a higher risk level would demand alternative wages at least as high in order to remain indifferent. As reflected in equation 1, this would follow from workers strictly preferring more wages and weakly preferring less risk.

Similar shape restrictions on indifference curves in attribute-price space will be credible in many settings where interest centers on WTP and hedonic analysis is commonly used. If utility increases in the attribute - sunshine as in ? or faster computer processors as in Pakes (2003) — rather than decreases, $\theta_{n}$ would decrease in the attribute level rather increase. Analogous results can be derived in that setting.

Substantively, C1.b means that as the risk level increases, workers require weakly increasing amounts of wage compensation to remain indifferent to a marginal change in risk levels. It is equivalent to a marginal rate of substitution that weakly increases in $r$. C1.b follows from preferences convex on $R \times W$. C1.b is a nontrivial assumption but plausible in many settings. Many have used it in the past including Rosen (1986), Pratt and Zeckhauser (1996), and Chay and Greenstone (2005) who use this kind of "weak restriction"

\footnotetext{
${ }^{3} U_{n}$ continuously differentiable in wages and twice continuously differentiable in risk would imply this.
} 
in their test for selection bias due to assortative matching. Consider an expected utility maximizer with

$$
U_{n}(r, w)=(1-r) u(w)+r v(w)
$$

where $u$ is the state-dependent utility if the worker does not suffer a fatal injury and $v$ the utility if so injured. Then, $\theta_{n}$ is convex if and only if $u^{\prime}>v^{\prime}$, that is if the marginal utility of wages is lower in the case of a fatal injury than in the case without. ${ }^{4}$

Prior research on state-dependent preferences for occupational risks are consistent with the idea that preferences are commonly convex. The estimates from the structural model of worker preferences in Biddle and Zarkin (1988) suggest that the vast majority of individuals have convex income-risk preferences. Viscusi and Evans (1990) estimate state-dependent utility functions with regard to nonfatal occupational injuries under various parametric specifications. In each case, their estimates suggest that preferences are convex on average. C1.b assumes they are convex for every individual.

In addition to increasing, convex indifference curves, informative partial identification requires that the attribute-price space under consideration is finitely bounded above. This upper bound provides a necessary fulcrum against which the shape restrictions on $\theta_{n}$ gain leverage for identification. The basic idea is illustrated in Figure 1 on page 8. C1-2 bound individual- $n$ 's status-quo indifference curve $\theta_{n}$ within the unshaded region. Proposition 1 expresses these bounds.

Condition 2. Risk-wage space $R \times W$ is finitely bounded above

$$
\forall r_{U}<\infty, \exists w_{U}<\infty \text { such that } w_{U} \geq \max _{n \in N} \theta_{n}\left(r_{U}\right)
$$

In the analysis, $\left(r_{U}, w_{U}\right)$ must be chosen as parameters and the two values must be understood together. Begin by considering a given risk level higher than all observed risk levels. Call it $r_{U}$. Then, $w_{U}$ will correspond to an upper-bound on wages that any worker would require to accept $r_{U}$ and remain indifferent relative to the status quo job type she is observed to have chosen.

If attention is restricted to moderate risk levels (like those found in workplaces rather than war zones), the idea of a finite upper bound $w_{U}$ is plausible. Wages could be made high enough to make any clerical worker $(r=0.5$ fatalities per 100,000 workers) indifferent to increasing her fatal occupational risk level to that of a construction worker $(r=12.9)$ [of Labor Statistics (2005)]. However, there may not be wages high enough to induce her to accept the risks faced by U.S. military personnel in Iraq $(r=392)$ [Preston and Buzzell (2006)].

To understand sensitivity in the application, estimation under a range of assumed values will be carried out. For instance, the least conservative estimate presented sets $r_{U}$ equal to 250 annual fatalities per 100,000 workers per year. This is chosen to be 10 percent higher than the highest risk level observed in the data: 226 for commercial fishers. The upper bound on wages $w_{U}$ is set at $\$ 107$ per hour, also 10 percent higher than the maximum wage observed in the data.

\footnotetext{
${ }^{4} \theta^{\prime}=\frac{u-v}{(1-r) u^{\prime}+r v^{\prime}}$ so $\left.\theta^{\prime \prime}=(u+v)[(1-r)) u^{\prime}+r v^{\prime}\right]^{-2}\left(u^{\prime}-v^{\prime}\right)$. The first two terms are positive. $\theta^{\prime \prime}$ takes the sign of $u^{\prime}-v^{\prime}$.
} 


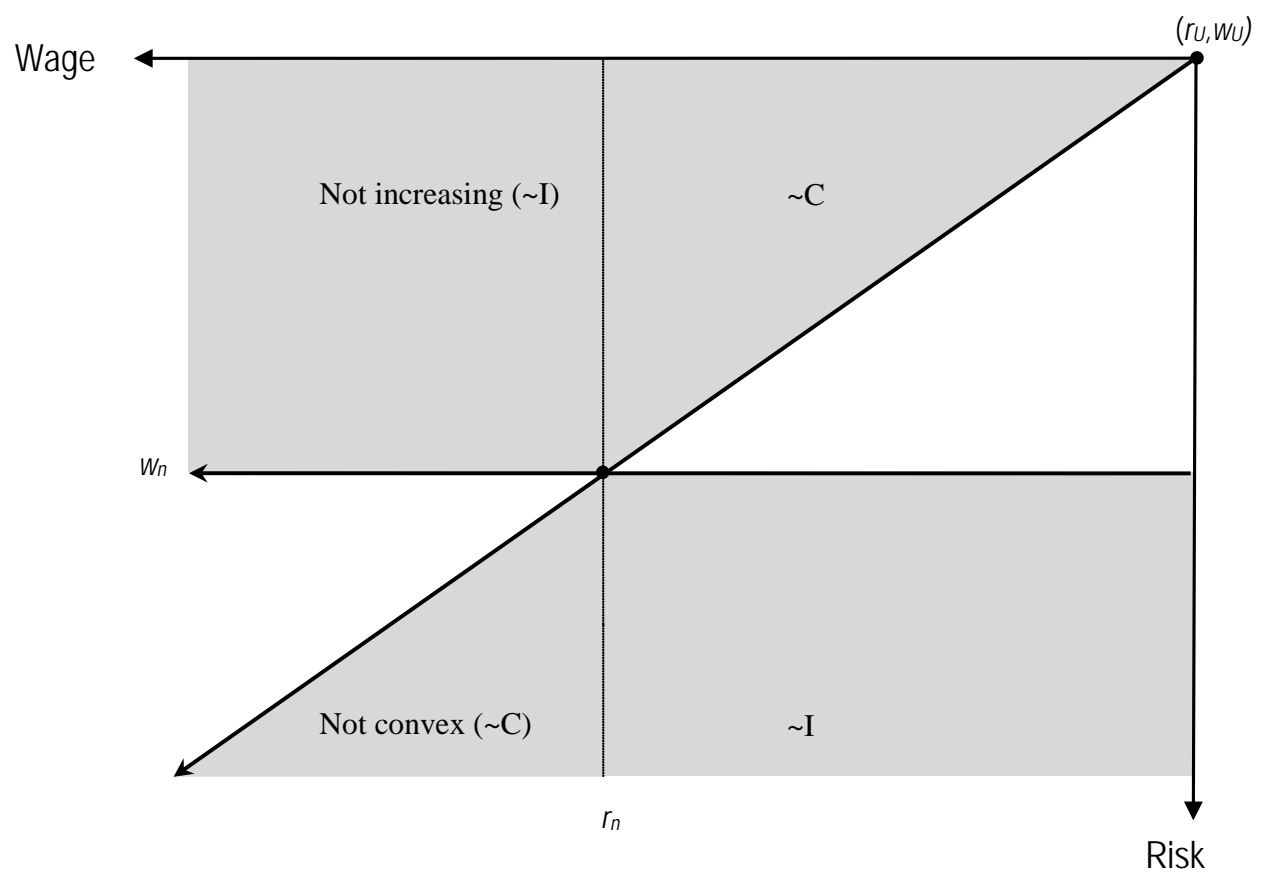

Figure 1: Bounds on increasing, convex indifference curve $\theta_{n}$ passing through $\left(r_{n}, w_{n}\right)$. 


\subsection{Partial identification of AWTP with shape restrictions alone}

This section studies the identifying power of the shape restrictions C1-2 alone for AWTP. The approach draws from the analysis of shape-restricted treatment-response functions in section 8.4 of Manski (2003). In the present analysis, risk levels are the "treatment" and indifference curves are the response functions. Identification of AWTP proceeds in three steps, each centered around a proposition. Proposition 1 describes how $C 1$ and $C 2$ impose bounds on any individual's indifference curve, $\theta_{n}$. Bounds on $\theta_{n}$ imply bounds on its slope at any point, $\theta_{n}^{\prime}$, expressed in Proposition 2. Taking expectations across individuals gives bounds on AWTP, $E \theta^{\prime}$, expressed in Proposition 3.

Step 1: Bound $\theta_{n}(\cdot)$ The individual indifference curve, $\theta_{n}$, must lie in the unshaded region of Figure 1. $\theta_{n}$ passes through $\left(r_{n}, w_{n}\right)$ by definition. Increasingness rules $\theta_{n}$ out of the two rectangular regions labeled ${ }^{\sim} \mathrm{I}$ in Figure 1. For $s<r_{n}$, if $\theta_{n}$ took values above $w_{n}$ it would have to decrease to attain $\left(r_{n}, w_{n}\right)$ violating increasingness. For $r>r_{n}$, it would have to decrease to take values lower than $w_{n}$.

Convexity rules $\theta_{n}$ out of the two triangular ${ }^{\sim} \mathrm{C}$ regions. Suppose the function passed through any point in a ${ }^{\sim} \mathrm{C}$ region. In order to also pass through $\left(r_{n}, w_{n}\right)$ and to remain increasing and convex, then $\theta_{n}\left(r_{U}\right) \geq w_{U}$, which would contradict C2. Note that convexity gives useful restrictions only when $r_{U}$ and $w_{U}$ are finite, which motivated $\mathrm{C} 2$.

Proposition 1 formally describes the bounds on $\theta_{n}$ generated by C1-2. It describes the interval of wage values within which an increasing, convex indifference curve must lie at a given risk level $s \in R$.

Proposition 1. Bound on indifference curve $\theta_{n}$ Given $C 1-2, L B_{1}(s) \leq \theta_{n}(s) \leq U B_{1}(s)$ for $\forall s \in R$ with

\begin{tabular}{l|l|l}
$L B_{1}(s) \equiv$ & $U B_{1}(s) \equiv$ & \\
\hline$w_{n}$ & $w_{n}+\frac{w_{U}-w_{n}}{r_{U}-r_{n}}\left(s-r_{n}\right)$ & if $s>r_{n}$ \\
$w_{n}+\frac{w_{U}-w_{n}}{r_{U}-r_{n}}\left(s-r_{n}\right)$ & $w_{n}$ & if $s<r_{n}$
\end{tabular}

These bounds are strict.

Proof. Consider the cases separately.

$s>r_{n}$ : $C 1$.a gives the lower bound, $\theta_{n}(s) \geq \theta_{n}\left(r_{n}\right)=w_{n}$. The upper bound is obtained by applying Lemma 9 with $r_{U} \equiv t$ and $r \equiv s .{ }^{5}$

$s \leq r_{n}$ : Lemma 10 with $s<r_{n}<r_{U}$ gives

$$
\theta_{n}(s) \geq \theta_{n}\left(r_{n}\right)+\frac{\theta_{n}\left(r_{U}\right)-\theta_{n}\left(r_{n}\right)}{r_{U}-r_{n}}\left(s-r_{n}\right)
$$

To obtain the desired lower bound, use $w_{n}=\theta_{n}\left(r_{n}\right)$ and, by $\mathrm{C} 2, w_{U} \geq \theta_{n}\left(r_{U}\right)$ noting that $\left(s-r_{n}\right)<0$. The upper bound is from $C 1 . a: \theta_{n}(s) \leq \theta_{n}\left(r_{n}\right)=w_{n}$.

\footnotetext{
${ }^{5}$ Lemmas are in the Appendix.
} 
Given these bounds on $\theta_{n}$, its slope is also bounded.

Step 2: Bound $\theta_{n}^{\prime}$ The lower and upper bounds on individual WTP are illustrated in Figure 2 and stated formally in Proposition 2. The slope at risk level $s$ is always maximized by assuming that the function $\theta_{n}$ is at its lower bound at $s$.

If $s>r_{N}$, the upper bound on the slope is $\frac{w_{U}-w_{n}}{r_{U}-s}$. This is consistent with $\theta_{n}$ constant at value $w_{n}$ for all risk levels below $s$, then linear from $\left(s, w_{n}\right)$ to $\left(r_{U}, w_{U}\right)$. This represents a very strange preference - someone indifferent to additional risk up to $s$ and abruptly averse with a constant marginal rate of substitution for additional risk above $s$. However, it is an extreme consistent with $\mathrm{C} 1-2$.

If $s<r_{n}$, the upper bound on the slope is $\frac{w_{U}-w_{n}}{r_{U}-r_{n}}$. Any greater slope would violate convexity.

In every case, the lower bound on the slope is given by increasingness, $\theta_{n}^{\prime}(s) \geq 0$. This cannot be tightened without further assumption. The lower bound is always zero because we have nothing to rule out a person who is indifferent to additional risk. So, indifference curves could be flat.

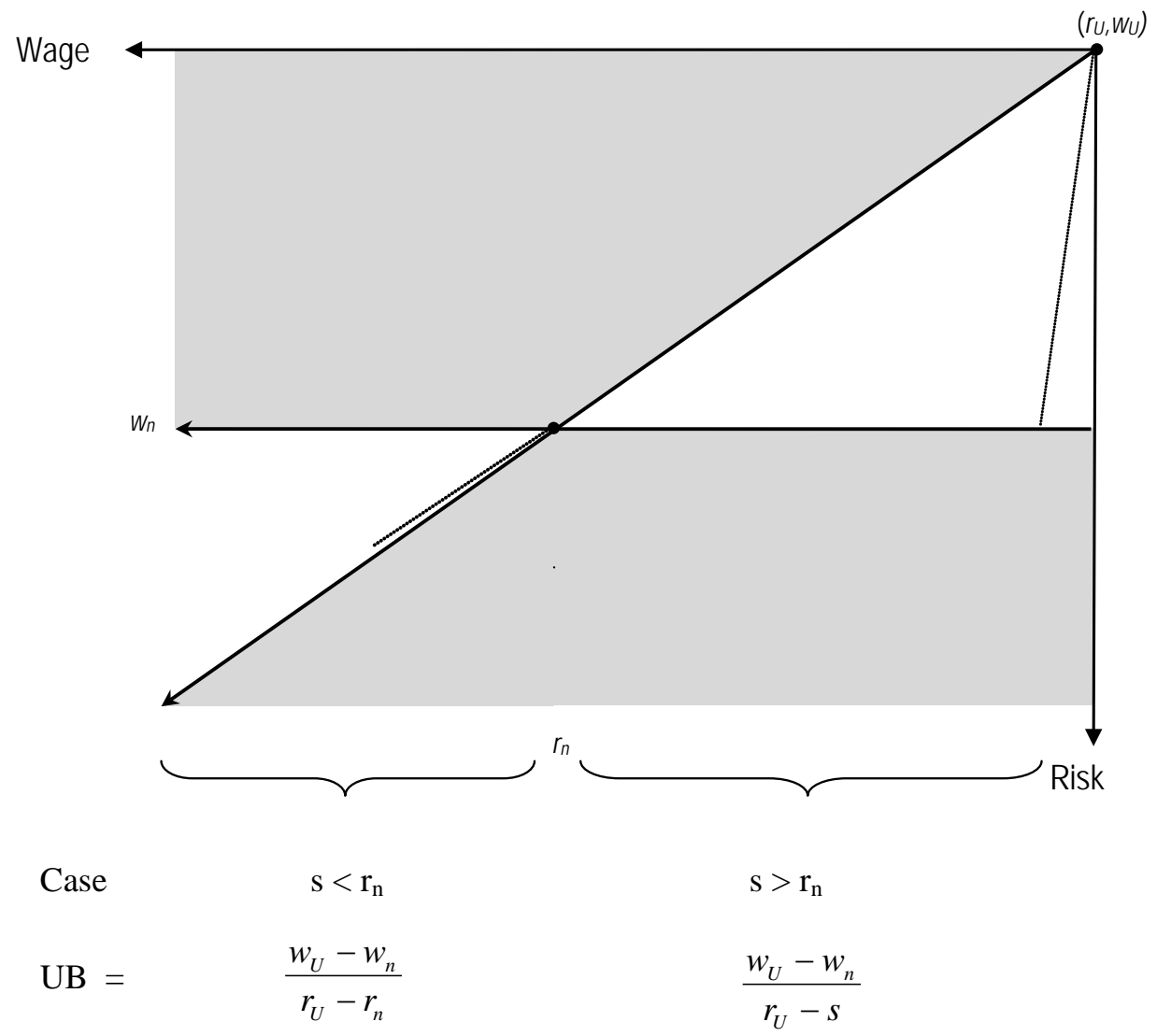

Figure 2: Upper bound on individual WTP $\left(\theta_{n}^{\prime}\right)$ by case 
Proposition 2. Bounds on $\theta_{n}^{\prime}$

Given C1-2, for $\forall s \in R$

$$
0 \leq \theta_{n}^{\prime}(s) \leq \frac{w_{U}-w_{n}}{r_{U}-s} 1\left[s>r_{n}\right]+\frac{w_{U}-w_{n}}{r_{U}-r_{n}} 1\left[s \leq r_{n}\right]
$$

These bounds are strict.

Proof. In each case, the slope at $s$ is maximized by assuming $\theta_{n}(s)$ is at its lower bound. In case $s \geq r_{N}$, suppose $\theta_{n}^{\prime}(s)>\frac{w_{U}-w_{n}}{r_{U}-s}$. For $t \in\left(s, r_{U}\right), \theta_{n}^{\prime}(t) \geq \theta_{n}^{\prime}(s)>\frac{w_{U}-w_{n}}{r_{U}-s}$ by convexity. Therefore,

$$
\begin{aligned}
\theta_{n}\left(r_{U}\right) & >\theta_{n}(s)+\frac{w_{U}-w_{n}}{r_{U}-s}\left(r_{U}-s\right) \\
& =\theta_{n}(s)+w_{U}-w_{n} \\
& \geq w_{U}
\end{aligned}
$$

This contradicts the definition of $w_{U}$ as an upper bound on $\theta_{n}$ proving $\theta_{n}^{\prime}(s) \leq \frac{w_{U}-w_{n}}{r_{U}-s}$ for $s \geq r_{n}$. The first inequality comes from the assumed conditions and by noting that a linear function is the minimal convex function. The third line comes from Proposition 1's lower bound on $\theta_{n}(s)$. The logic of the other case proceeds analogously.

Step 3: Bound on $E \theta^{\prime}$ To obtain bounds on the AWTP, take the expectation of the bounds with respect to the distribution of the population of indifference curves. This gives the following proposition.

Proposition 3. Bounds on AWTP, E $\theta^{\prime}$

Given C1-2, for $\forall s \in R$,

$$
\begin{gathered}
L B_{3}(s)=0 \leq E\left[\theta_{n}^{\prime}(s)\right] \\
\leq E\left[\frac{w_{U}-w_{n}}{r_{U}-s} \mid s>r_{n}\right] \operatorname{Pr}\left[s>r_{n}\right]+E\left[\frac{w_{U}-w_{n}}{r_{U}-r_{n}} \mid s \leq r_{n}\right] \operatorname{Pr}\left[s \leq r_{n}\right] \equiv U B_{3}(s)
\end{gathered}
$$

These bounds are strict.

Proof. Take expectation of Proposition 2's bound on $\theta_{n}^{\prime}$ across the population of individuals.

The sample analogue of this expression estimates the upper bound on the AWTP at any risk level $s \in R$. Given parameters $\left(r_{U}, w_{U}\right)$ and data $\left(r_{n}, w_{n}\right)$ for $n=1,2 \ldots N$, each observation falls into either $s>r_{n}$ or $s \leq r_{n}$. Then, estimate the upper bound on the average slope at risk level $s$ with:

$$
\hat{U} B_{3}(s)=N^{-1} \sum_{n}\left(\frac{w_{U}-w_{n}}{r_{U}-s} 1\left[s>r_{n}\right]+\frac{w_{U}-w_{n}}{r_{U}-r_{n}} 1\left[s \leq r_{n}\right]\right)
$$

Some comparative statics illuminates how the Proposition 2 upper bound on WTP, $U B_{3}$, depends on parameters. The logic of AWTP is the same. 
- Increasing $w_{U}$ strictly increases the bound $U B_{3}$. Formally, this increases the numerator in either case. Economically, increasing $w_{U}$ says that workers may demand higher wages to remain indifferent between their status quo and a job with risk level $r_{U}$, loosening the limit on the levels to which compensating differentials can rise and, thereby, loosening the rate at which they can rise.

- Increasing $r_{U}$ strictly decreases the bound. Increases denominator. Economically, this raises the risk level at which workers must be willing to accept a fixed $w_{U}$ in exchange for the status quo. It rules out the highest levels of distaste for risk that were consistent with a lower $r_{U}$.

- Upper bound weakly increases in risk level. For $s, t \in R$ with $s<t, U B_{3}(s) \leq U B_{3}(t)$. This could happen for two reasons: case switching and increases within the $r_{n}<s<t$ case. Holding parameters and data fixed, the upper bound on $\theta_{n}^{\prime}(s)$ increases as the case changes from $s \leq r_{n}$ to $r_{n}<s$. This is illustrated by the increasing slopes of the dotted lines between cases in Figure 2. Therefore, if $s$ and $t$ are in different cases, $U B_{3}(s)<U B_{3}(t)$. If $r_{n}<s<t$, then $U B_{3}(s)<U B_{3}(t)$ because $\frac{w_{U}-w_{n}}{r_{U}-s}<\frac{w_{U}-w_{n}}{r_{U}-t}$. If $s<t<r_{n}$, then $U B_{3}(s)=U B_{3}(t)$.

This has important implications for the econometrician's choice of parameters $\left(r_{U}, w_{U}\right)$. The upper bound on WTP and AWTP at any given risk level $s$ can be made arbitrarily large by setting $r_{U}$ to make $\left(r_{U}-s\right)$ arbitrarily small. This is true given any data and assuming finite $w_{U}$. For such a choice, all observations are in case $s>r_{n}$ and the bound on all $\theta_{n}^{\prime}(s), \frac{w_{U}-w_{n}}{r_{U}-s}$, is made arbitrarily large. If research interest focuses on some particular risk level, such as median or mean level of risk, one will want to set $r_{U}$ well above this level and set $w_{U}$ given that choice.

\subsection{Modeling the market}

This section integrates the results developed above using only shape restrictions on statusquo indifference curves with a conventional hedonic model of the market defining individual's opportunities. This models each worker's choice set, among which the observed job is assumed preferred. Choice sets are equilibrium objects arising from the interaction of workers and firms and are characterized by a pricing function for hedonic attributes given worker characteristics. In the case considered here, the pricing function maps job and worker characteristics to maximum wage offers.

The focus is not on identification of the price functions but on how knowledge of the price function interacts with $\mathrm{C} 1$ and $\mathrm{C} 2$ to generate new restrictions on indifference curves $\theta_{n}$ and, thereby, to generate tighter bounds on individual WTP, $\theta_{n}^{\prime}$, and AWTP, $E \theta_{n}^{\prime}$. Two classes of pricing functions are considered, as in Bajari and Benkard (2005). The first is general, allowing for discrete, discontinuous price functions. The second class is restrictive, allowing wages to vary only smoothly and continuously with risk. This characterizes the approach in most of the empirical VSL and broader hedonics literature.

First, we consider a pricing function that allows for a finite number of opportunities for any individual worker. Knowledge of the pricing function generates new restrictions on 
indifference curves by revealed preference. In combination with $\mathrm{C} 1-2$, this implies tighter bounds than either shape restrictions alone (Propositions 1-3) or the price function alone, as studied in Pakes (2003), give. Propositions 4 and 5 describe the tighter bounds so obtained on $\theta_{n}$ and $E \theta^{\prime}$ respectively.

Second, we consider the implications of a known smooth, continuous price function for identification of WTP and AWTP. This type of market implies a tangency condition between the price function and the status-quo indifference curve at the observed job. Most conventional hedonic analysis uses this condition. Under $\mathrm{C} 1-2$, the tangency condition tightens the bounds further but does not point identify preferences, WTP or AWTP.

This is interesting for two reasons. First, it is valuable to understand the identifying power of this condition in order to estimate bounds on AWTP and VSL given our structure. Second, it is interesting to see that AWTP is not point identified by the tangency condition in a conventionally-defined, well-behaved market foregoing functional form restrictions on utility.

Thus far, all worker and most job characteristics have been kept buried in the heterogeneity of $U_{n}$ and $\theta_{n}$, as we studied only WTP for ceteris paribus changes in risk level. In order to capture differences in the opportunities available to workers, the influence of observed and unobserved worker and job characteristics are modeled.

Let $a \in A \subseteq \mathbf{R}^{n_{a}}$ measure observable characteristics of the job (other than risk) that affect wage. This might include measures of tasks required and working conditions. The econometrician now characterizes jobs by $(r, a, w)$. Similarly, let $x \in X \subseteq \mathbf{R}^{n_{x}}$ measure observable characteristics of workers that affect potential wages, for instance education. Workers also have productive heterogeneity $v \in H \subseteq \mathbf{R}$, which is known to workers and firms and influences wages, but which the econometrician does not observe. Utility is now $U_{n}(r, a, w), \theta_{n}$ implicitly defined holding $a=a_{n}$, and data are $\left\{\left(r_{n}, a_{n}, w_{n}, x_{n}\right)\right\}_{n=1}^{N}$.

\subsubsection{General opportunity sets}

Each worker $(x, v)$ takes a set of available market opportunities as given and chooses a job optimally from it. This set can be expressed as a function mapping any available job type $(r, a)$ to the maximum available wage for that worker type. For any worker, her market opportunity set is $P: R \times A \times X \times H \rightarrow W$ defined by $w=P(r, a, x, v)$. This price function is an equilibrium object arising out of the interactions of workers and firms. Workers are assumed to be price takers for whom $P$ is fixed. No particular industrial structure is assumed on the firm side of the market. The price function may be discrete or discontinuous. Since all preferences are monotone in wage and risk, $P$ must be weakly monotone increasing in $r$ or it could not be an equilibrium.

Assume that $P(r, a ; x, v)$ and $v_{n}$ are identified.

Condition 3. Each worker chooses an offer optimally from an identified $P(r, a, x, v)$ which clears the market and each worker's $v_{n}$ is identified.

Identification is not trivial but the problem is well understood and can be addressed in various ways depending on the setting. The problem of identifying $P$ and $v_{n}$ can often be separated from the problem of identifying features of preferences given that information. Bajari and Benkard (2005) discuss approaches to identifying $P$. One common 
set of assumptions is $E[v \mid r, a, x] \equiv 0$ and $P(r, a ; x, v) \equiv e^{H(r, a ; x)+v}$. This identifies $P$ and rules out selection bias in estimation of its unknown features. ${ }^{6}$ It also implies that $v_{n}=\ln \left(w_{n}\right)-H\left(r_{n}, a_{n} ; x_{n}\right)$. An even more specific version of this will be assumed later for the empirical analysis.

By revealed preference, choice of job $\left(r=r_{n}, a=a_{n}\right)$ by worker $\left(x=x_{n}, v=v_{n}\right)$ implies that her status-quo indifference curve $\theta_{n}(\cdot)$ is bounded below in risk-wage space by the price function $P\left(\cdot, a_{n} ; x_{n}, v_{n}\right)$. Otherwise, the worker could attain higher utility by choosing a different job. For convenience, we can condition on any individual's characteristics and chosen amenities, suppress those variables and consider only the relationship between her indifference curve $\theta_{n}$ and price function $P_{n}(\cdot) \equiv P\left(\cdot, a_{n} ; x_{n}, v_{n}\right)$.

C3 implies $\theta_{n}\left(r_{n}\right)=P_{n}\left(r_{n}\right)$ and $\forall s \in R$,

$$
P_{n}(s) \leq \theta_{n}(s)
$$

This implies that model would be internally inconsistent if there existed $s$ such that $U B_{1}(s)<P_{n}(s)$, as Proposition 1 showed that $\theta_{n}(s) \leq U B_{1}(s)$. Given $P$, this implies a lower bound on valid values of $w_{U}$, call it $w_{l u b}$. One can choose $w_{U}$ high enough, which raises $U B_{1}$, to insure that this does not occur. In the application, this is done given $\hat{P}$.

The propositions from this section are not used in the empirical analysis but provide a stepping stone to the study of continuous price functions and a set of theoretical restrictions that could prove useful in other contexts. Combining inequality (3), which follows from C3, with C1-2 tightens the lower bound on $\theta_{n}$. The same kind of logic is used again although the details differ.

The new lower bound on $\theta_{n}$ provided by $P_{n}$ gives new fulcrums against which the leverage of the shape restrictions $C 1-2$ can be exploited. For instance, information that $P_{n}$ takes values $P_{n}(q)$ and $P_{n}(t)$ newly rules $\theta_{n}$ out of the striped regions illustrated in Figure 3. Considering the logic of this figure can give useful insight into this source of identification. If $\theta_{n}$ were to take values in the diagonally-striped regions, $\theta_{n}$ would have to violate $\mathrm{C} 1$ or $\mathrm{C} 2$. For $s<q, \theta_{n}(s)$ in the striped region violates convexity by logic exactly analogous to that which gave a lower bound on $\theta_{n}$ in Proposition 1 for $s<r_{n}$. If $\theta_{n}$ were to take a value in the striped region it would need a steep slope to attain $\theta_{n}(q) \geq P_{n}(q)$ followed by a shallower slope to attain $\theta_{n}\left(r_{n}\right)=w_{n}$, violating convexity. For $s \in\left(r_{n}, t\right)$, $\theta_{n}(s)$ in the striped region violates convexity by a similar logic. For $s \in\left[q, r_{n}\right), \theta_{n}(s)$ in the striped region violates increasingness as $P_{n}(q) \leq \theta_{n}(q) \leq \theta_{n}(s)$. For $s>t, \theta_{n}(s)$ in the

\footnotetext{
${ }^{6}$ Credibly satisfying this condition when using hedonics to study VSL is challenging due to potential correlations between workers' tastes for risk and either their general or risk-reducing skills (Brown (1980); Hwang, Reed, and Hubbard (1992); Shogren and Stamland (2002)). For instance, unobserved productivity may be negatively correlated with taste for risk so that people observed in low risk jobs will also tend to have higher unobserved productivity. This may lead to an underestimate of the market price for risk and of VSL. A second problem may also arise especially in the hedonic analysis of labor markets, where income is determined. Consider two workers who differ only in $v$. At any particular job $(r, a)$, one would be offered higher wages, which could lead to higher income. If job safety is a normal good, then that worker may choose a lower risk job inducing a negative correlation between observed $r$ and unobserved $v$ and violating $E[v \mid r, a, x]=0$. Despite these problems, such conditions are commonly maintained and are maintained here.
} 
striped region violates convexity because it requires $\theta_{n}^{\prime}$ to decrease somewhere between $t$ and $s$.

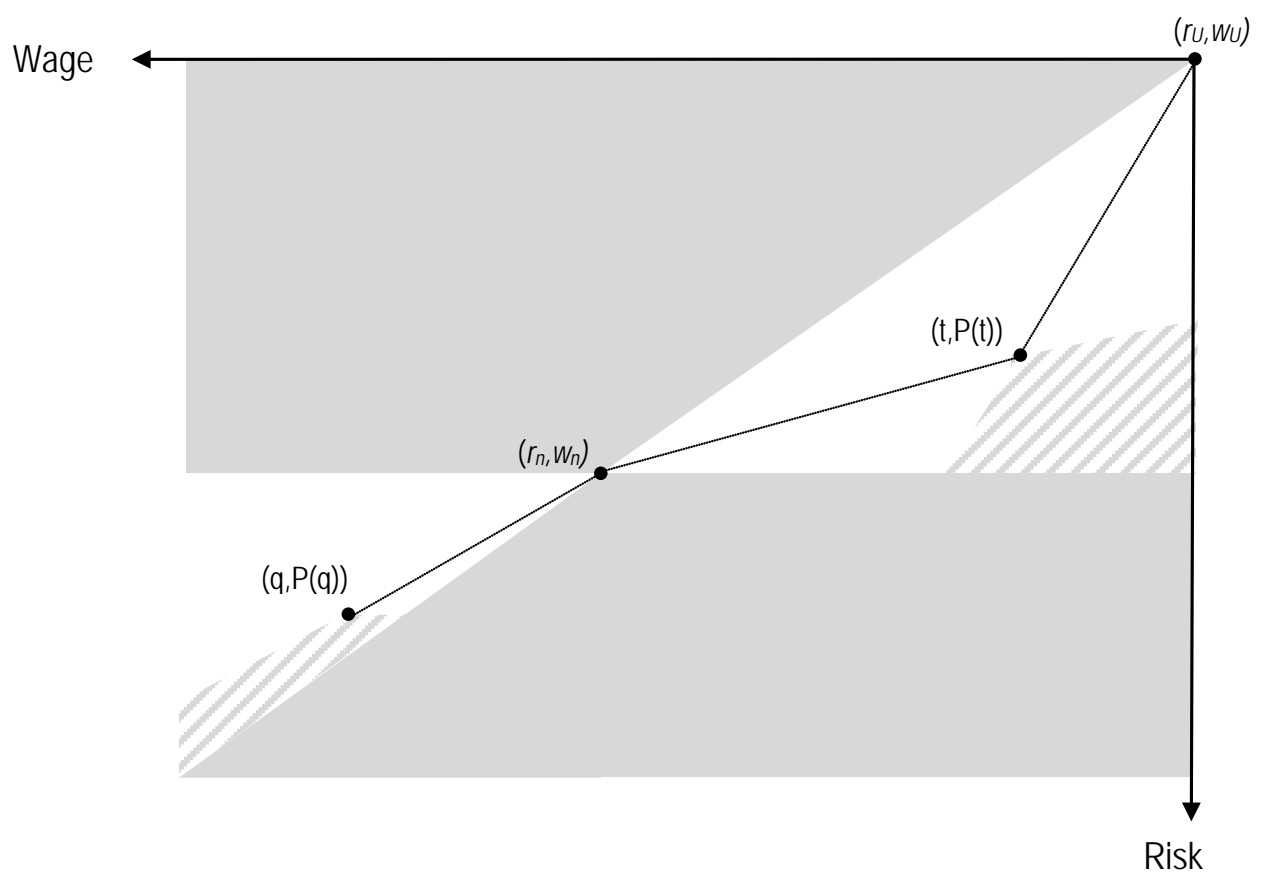

Figure 3: Bounds on $\theta_{n}$ given knowledge of two points on $P_{n}$ below $\theta_{n}$

The mathematical expressions for the bounds (Propositions 4 and 5) are complicated but they formalize the idea in this figure and generalize from information about $P_{n}$ at the two points $q$ and $t$ to information about $P_{n}$ across any set of risk levels. Translating information about $P_{n}$ at many values into a bound on $\theta_{n}(s)$ for any particular $s$ requires searching over appropriate ranges in $R$ to see which constraints bind most tightly at a given risk level of interest $s$. They tighten the lower bound on $\theta_{n}$.

Proposition 4. Given C1-3, for any $s \in R$

$L B_{4}(s) \leq \theta_{n}(s) \leq U B_{1}(s)$ with $L B_{4}(s)$ defined as 


$$
\begin{gathered}
\max \left\{\begin{array}{c}
L B_{1}(s), \\
w_{n}+\max _{\rho \in\left(r_{n}, s\right)} \frac{P_{n}(\rho)-P_{n}\left(r_{n}\right)}{\rho-r_{n}}\left(s-r_{n}\right), \\
\max _{\tau \in\left(s, r_{U}\right)} P_{n}(\tau)+\frac{w_{U}-P_{n}(\tau)}{r_{U}-\tau}(s-\tau)
\end{array}\right\} \quad \text { if } s>r_{n} \\
\max \left\{\begin{array}{c}
L B_{1}(s), \\
w_{n}+\min _{q \in\left(s, r_{n}\right)} \frac{P_{n}\left(r_{n}\right)-P_{n}(q)}{r_{n}-q}\left(r_{n}-s\right), \\
\max _{\tau \in\left(r_{L}, r\right)} P_{n}(\tau)
\end{array} \quad \text { if } s<r_{n}\right.
\end{gathered}
$$

Proof. For $s>r_{n}$, the lower bound on $\theta_{n}(s)$ is the maximum across three arguments. The first argument comes from the lower bound in Proposition 1. The second comes from searching across all values of risk $\rho$ between $r_{n}$ and $s$ for identification as pictured in the far right of Figure 3 and proved in Lemma 11. The third argument is from searching across all values of risk $\tau$ between $s$ and $r_{U}$ for identification as pictured in the $\left(r_{n}, s\right)$ region of Figure 3. To prove it holds, use Lemma 10 with $s<\tau<r_{U}$ to obtain

$$
\theta_{n}(s) \geq \theta_{n}(\tau)+\frac{\theta_{n}\left(r_{U}\right)-\theta_{n}(\tau)}{r_{u}-\tau}(s-\tau)
$$

Using C3: $P_{n}(\tau) \leq \theta_{n}(\tau)$ and C2: $\theta_{n}\left(r_{U}\right) \leq w_{U}$ gives the desired expression for any $\tau \in\left[s, r_{U}\right]$. Since this holds for any such $\tau$, the maximum across all such $\tau$ gives the tightest lower bound from this source of identification. The upper bound is from Proposition 1.

For $s<r_{n}$, the lower bound is again the maximum of three arguments. The first argument is from Proposition 1. The second argument is proved in Lemma 12 with $s \equiv p$. It exploits information about $P_{n}$ over values between $s$ and $r_{n}$ to achieve identification as pictured in Figure 3 at values below $q$. The third argument follows from $P_{n}(\tau) \leq \theta_{n}(\tau) \leq$ $\theta_{n}(s)$ for any and all $\tau<s$. The first inequality comes by $P_{n}$ bounding $\theta$ below. The second comes by $\theta_{n}$ increasing. It exploits information about $P_{n}$ at values below $s$ to achieve identification as pictured at values between $q$ and $r_{n}$. The upper bound is from Proposition 1.

Given these bounds on the individual indifference curves $\theta_{n}$, bounds on the slopes of the individual indifference curves $\theta_{n}^{\prime}$ and the average indifference curve across the population $E \theta_{n}^{\prime}$ can be obtained.

Proposition 5. Bounds on AWTP with discrete $P$

Given C1-3, for $\forall s \in R$

$$
\begin{aligned}
& E\left[\frac{L B_{4}(s)-w_{n}}{s-r_{n}} \mid s>r_{n}\right] \operatorname{Pr}\left[s>r_{n}\right] \leq E\left[\theta_{n}^{\prime}(s)\right] \\
\leq & E\left[\frac{w_{U}-L B_{4}(s)}{r_{U}-s} \mid s>r_{n}\right] \operatorname{Pr}\left[s>r_{n}\right]+E\left[\frac{w_{n}-L B_{4}(s)}{r_{n}-s} \mid s \leq r_{n}\right] \operatorname{Pr}\left[s \leq r_{n}\right]
\end{aligned}
$$


Proof. For the lower bound, individual WTP $\theta_{n}^{\prime}$ could be 0 for $s<r_{n}$. For $s \geq r_{n}$, the minimum slope is given by that of a line from $\left(r_{n}, w_{n}\right)$ to $\left(s, L B_{4}(s)\right)$. Any lower value would violate Proposition 4 . Any higher value would not be a lower bound.

For the upper bound, individual WTP $\theta_{n}^{\prime}$ are maximized by searching across $t>s$ to minimize $\frac{U B_{4}(t)-L B_{4}(s)}{t-s}$. Any higher values for this slope would violate C1.b or C2. Any lower values is not the upper bound. For $s>r_{n}$, the relevant $t$ is $r_{U}$, which gives $U B_{4}(t)=$ $U B_{4}\left(r_{U}\right)=w_{U}$. For $s<r_{n}$, the relevant $t$ is $r_{n}$, which gives $U B_{4}(t)=U B_{4}\left(r_{n}\right)=w_{n}$.

Taking the expectation across individuals' $\theta_{n}^{\prime}$ bounds gives the bound on AWTP.

With this expression and information about a discrete price function $P$, one can compute bounds on AWTP and, hence, on VSL. However, in this application, we will assume a continuous price function. This generates another restriction, which is integrated into the analysis in the next section.

\subsubsection{Continuous price functions}

With a few exceptions (Heckman, Matzkin, and Nesheim (2003); Pakes (2003); Pakes (2008); Bajari and Benkard (2005)), most hedonic analyses assume a continuous price function. At each point, its slope is the market price for accepting marginally more risk. Optimal choices by each worker implies a tangency condition between the individual indifference curve and the market price curve at the observed job - the individual chooses a job which sets her marginal rate of substitution of money for risk equal to the market price of risk. This identifies $\theta_{n}^{\prime}\left(r_{n}\right)=P_{n}^{\prime}\left(r_{n}\right)$. In most hedonic analysis, enough structure is imposed on preferences to point-identify preferences from this tangency condition. This requires convenient assumptions about the known functional form of utility. In the current structure $(C 1-C 3)$, tangency generates new restrictions but still does not produce point identification. It will tighten the bounds on $\theta_{n}(\cdot)$ obtained previously, leading to yet tighter bounds on AWTP than Propositions 5 gave. Given an additional condition, concave $P_{n}$, identification and estimation are simplified considerably. As the data appear consistent with concave $P_{n}$, this will be exploited in the empirical analysis.

Consider a smoothness condition on $P$.

Condition 4. Smooth market price curve The quantity of workers supplied at all values of $(r, a, x, v)$ is positive (dense market) and $P$ is twice continuously differentiable in $r$, .

$C 4$ is a substantive assumption made here and elsewhere for theoretical and notational convenience. As Heckman, Matzkin, and Nesheim (2003) show, smoothness is not generally a property of market offer curves, even when workers' preferences and firms' profit functions are smooth. However, it ensures that the equilibrium market price of risk is well-defined as the partial derivative of $P$ with respect to $r$.

Utility maximization by workers implies that, at the observed job, each worker's status quo indifference curve is tangent to the market price curve. Each worker selects the job with risk and amenity level $\left(r^{*}, a^{*}\right)$ that maximizes $U_{n}\left(r^{*}, a^{*}, w^{*}\right)$ subject to wage offers $w^{*}=P\left(r^{*}, a^{*}, x_{n}, v_{n}\right)$. She chooses a job along the price curve that sets the marginal utility 
of wages times the marginal wage with respect to risk equal to the marginal disutility of risk. $^{7}$

The first-order condition is

$$
\frac{\partial U_{n}\left(r^{*}, a^{*}, w^{*}\right)}{\partial w} \cdot \frac{\partial P\left(r^{*}, a^{*} ; x_{n}, v_{n}\right)}{\partial r}+\frac{U_{n}\left(r^{*}, a^{*}, w^{*}\right)}{\partial r}=0
$$

For any worker- $n$, her observed job $\left(r_{n}, a_{n}, w_{n}\right)$ is revealed to be $\left(r^{*}, a^{*}, w^{*}\right)$, implying that the slope of the price function with respect to risk equals the slope of her status quo indifference curve at that point,

$$
m_{n} \equiv \frac{\partial P\left(r_{n}, a_{n} ; x_{n}, v_{n}\right)}{\partial r}=-\frac{\frac{\partial U_{n}\left(r_{n}, a_{n}, w_{n}\right)}{\partial r}}{\frac{\partial U_{n}\left(r_{n}, a_{n}, w_{n}\right)}{\partial w}}=\theta_{n}^{\prime}\left(r_{n}\right)
$$

First, $m_{n}$ is defined as the slope of the price function at individual- $n$ 's observed job. The next equality comes by rearranging the FOC. The third equality comes from equation (1). It is just a version of the regular tangency condition between an optimizing agent's indifference curve and her budget constraint. Figure 4 illustrates an optimizing worker's status $q u o$ indifference curve in risk-wage space tangent to the market-price curve $P$ conditional on a fixed $(a, x, v)$.

If $P$ were not smooth at $r_{n}$, if $\mathrm{C} 4$ did not hold, then the market price for a marginal change in risk would not be well-defined. While WTP would still be well-defined for workers observed at $r_{n}$ as a property of the indifference curve, it would no longer be identified by equation 4.

Equation 4 adds a new restriction to those embodied in Proposition 4 and 5. The basic idea is illustrated in Figure 5. Given that $\theta_{n}^{\prime}\left(r_{n}\right)=m_{n}, \theta_{n}\left(r_{n}\right)=P_{n}\left(r_{n}\right)=w_{n}$ and $\theta_{n}$ increasing and convex, then $\theta_{n}$ is bounded out of the region shaded with horizontal stripes. This shaded region is bounded above by a line passing through $\left(r_{n}, w_{n}\right)$ with slope $m_{n}$.

Proposition 6. Bounds on $\theta_{n}$ with smooth $P$

Given $C 1-4$, for any $s \in R$,

$\max \left\{L B_{4}(s), w_{n}+m_{n}\left(s-r_{n}\right)\right\} \leq \theta_{n}(s) \leq U B_{4}(s)$.

Proof. All the conditions generating the proposition 4 bounds still hold. To them, the tangency condition (equation 4) adds one more restriction. For $s>r_{n}\left(s<r_{n}\right)$, by convexity, the slope of the line connecting $\left(s, \theta_{n}(s)\right)$ to $\left(r_{n}, w_{n}\right)$ must be greater (less) than or equal to $m_{n}$ so $\frac{\theta_{n}(s)-w_{n}}{s-r_{n}} \geq m_{n}\left(\frac{w_{n}-\theta_{n}(s)}{r_{n}-s} \leq m_{n}\right)$. Rearranging yields the implication.

The individual slopes and AWTP are bound by the exact analogue of Proposition 5 with $\max \left\{L B_{4}(s), w_{n}+m_{n}\left(s-r_{n}\right)\right\}$ substituted for $L B_{4}$.

In general, any of these four conditions, three from $L B_{4}$ and one from the tangency condition, might be the binding lower bound for a given $s$. However, if market price is concave in risk, then the restriction generated by the tangency condition will necessarily bind more tightly than those newly-derived in the previous section and expressed in Proposition 4 because concave $P$ ensures that $P_{n}$ lies below the $w_{n}+m_{n}\left(s-r_{n}\right)$ line. This case is illustrated in Figure 5

\footnotetext{
${ }^{7} \mathrm{~A}$ second-order condition is also required: $\left(\frac{\partial^{2} U_{n}}{\partial w \partial r}+\frac{\partial^{2} U_{n}}{\partial^{2} w} \frac{\partial P}{\partial r}\right) \frac{\partial P}{\partial r}+\frac{\partial U_{n}}{\partial w} \frac{\partial^{2} P}{\partial^{2} r}+\frac{\partial^{2} U_{n}}{\partial^{2} r}+\frac{\partial^{2} U_{n}}{\partial r \partial w} \frac{\partial P}{\partial r}<0$.
} 


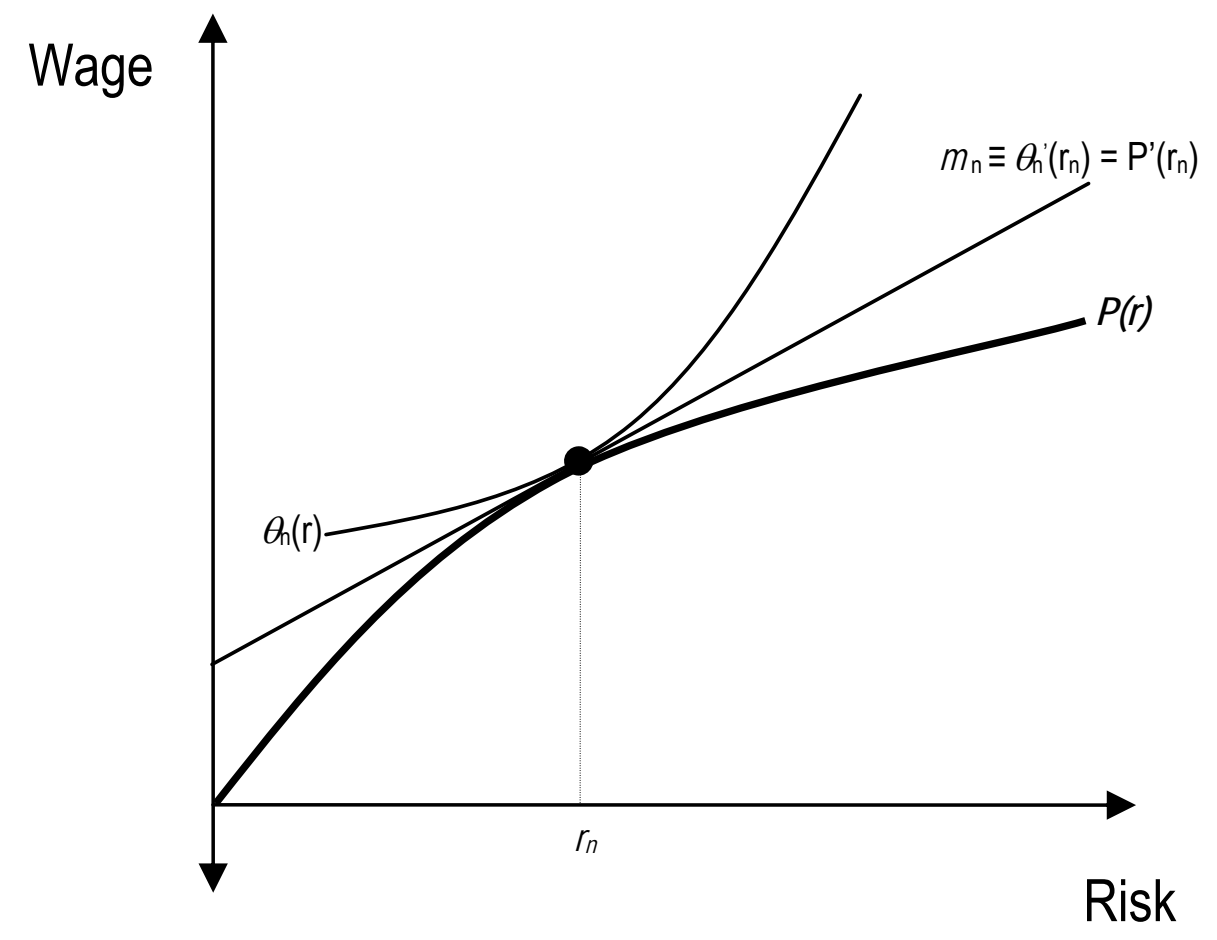

Figure 4: WTP at $r_{n}$ given smooth equilibrium price function 


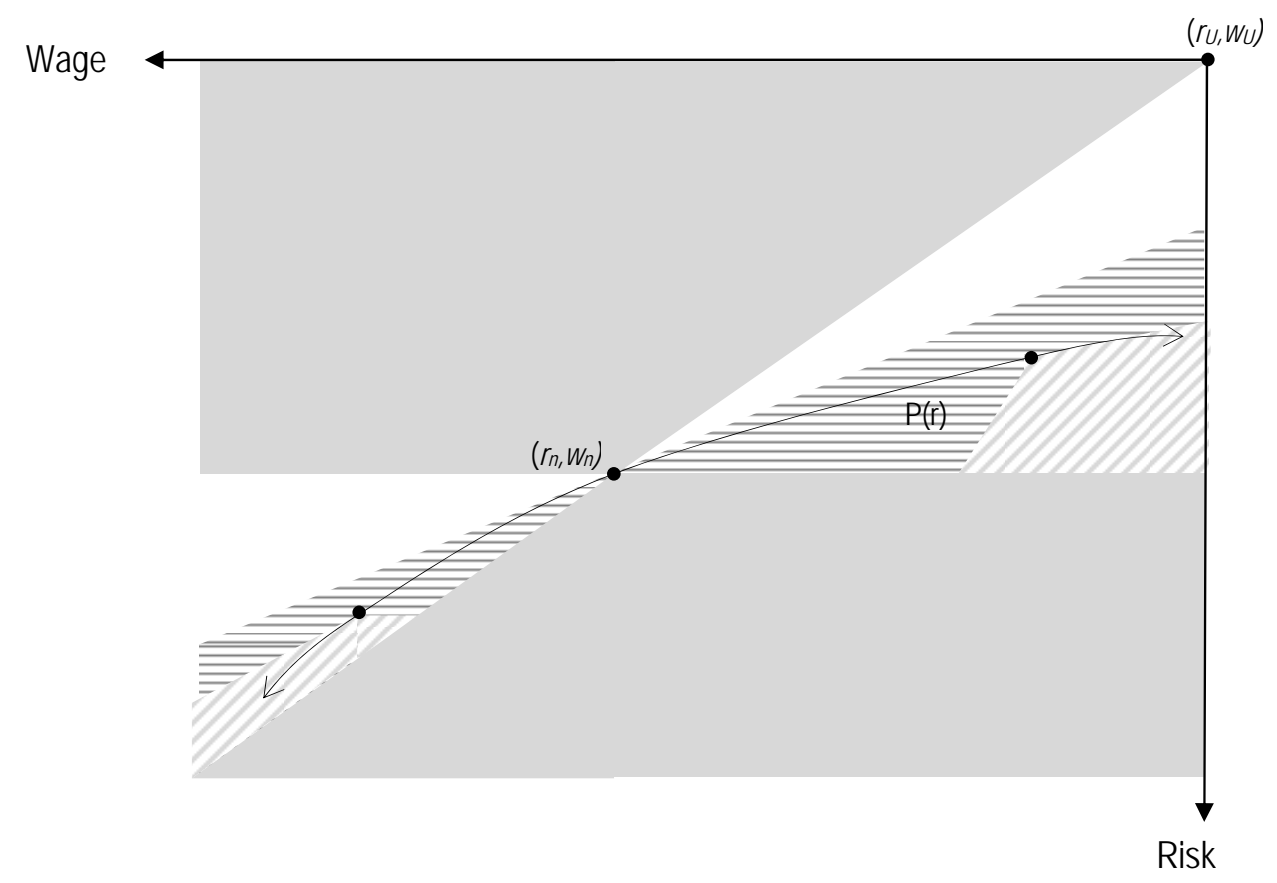

Figure 5: Bounds on $\theta_{n}$ given smooth equilibrium price function

Condition 5. $P$ concave in $R: \frac{P_{n}(s)-P_{n}(r)}{s-r} \geq \frac{P_{n}(t)-P_{n}(s)}{t-s}$ for $r<s<t$

Propositions 7 and 8 describe the bounds on $\theta_{n}$ and $E \theta^{\prime}$ respectively assuming smooth, concave $P$.

Proposition 7. Bounds on $\theta_{n}$ with smooth, concave $P$

Given $C 1-5$, for any $s \in R$

$\max \left\{L B_{1}(s), w_{n}+m_{n}\left(s-r_{n}\right)\right\} \leq \theta_{n}(s) \leq U B_{1}(s)$.

Proof. $P$ concave and nondecreasing implies that $m_{n}=P_{n}^{\prime}\left(r_{n}\right) \geq \frac{P_{n}(\rho)-P_{n}\left(r_{n}\right)}{\rho-r_{n}}$. This immediately implies that the new restriction binds tighter than the second restriction in Proposition 4 for $s>r_{n}$,

$$
w_{n}+m_{n}\left(s-r_{n}\right) \geq w_{n}+\max _{\rho \in\left(r_{n}, s\right)} \frac{P_{n}(\rho)-P_{n}\left(r_{n}\right)}{\rho-r_{n}}\left(s-r_{n}\right)
$$

Next, we prove that the new restriction binds tighter than the third restriction in Proposition 4. Note that $w_{U} \geq w_{\text {lub }}$ ensures that

$$
w_{U} \geq w_{n}+m_{n}\left(r_{U}-r_{n}\right)
$$

Also, given $w_{n}=P_{n}\left(r_{n}\right)$ and $m_{n}=P_{n}^{\prime}\left(r_{n}\right)$, at $\tau>r_{n}$, a concave price function must be below a linear function through $\left(r_{n}, w_{n}\right)$ with slope $m_{n}$, 


$$
P_{n}(\tau) \leq w_{n}+m_{n}\left(\tau-r_{n}\right)
$$

Substituting in inequalities 5 and 6 implies that,

$$
w_{U}-P_{n}(\tau) \geq w_{n}+m_{n}\left(r_{U}-r_{n}\right)-w_{n}-m_{n}\left(\tau-r_{n}\right)
$$

Rearranging implies,

$$
\frac{w_{U}-P_{n}(\tau)}{r_{U}-\tau} \geq m_{n}
$$

For $\tau>s>r_{n}$

$$
\begin{aligned}
P_{n}(\tau)+\frac{w_{U}-P_{n}(\tau)}{r_{U}-\tau}(s-\tau) & \leq P_{n}(\tau)+m_{n}(s-\tau) \\
& \leq w_{n}+m_{n}\left(\tau-r_{n}\right)+m_{n}(s-\tau) \\
& =\theta_{n}\left(r_{n}\right)+m_{n}\left(s-r_{n}\right)
\end{aligned}
$$

The first line comes from inequality 7 . The second from inequality 6 . The third line collects terms and uses $w_{n}=\theta_{n}\left(r_{n}\right)$. As this is true for any $\tau>s$, it is true for the maximum of all such $\tau$. This proves that the new restriction binds tighter than the third restriction of Proposition 4 for $s>r_{n}$. Similar arguments prove that the new restriction binds more tightly than the second and third restriction in that proposition for $s<r_{n}$.

This implies the following bounds on AWTP.

Proposition 8. Bounds on AWTP with smooth, concave $P$

Given C1-5, for any $s \in R$,

$$
\begin{aligned}
L B_{8}(s) \equiv & E\left[m_{n} \mid s \geq r_{n}\right] \operatorname{Pr}\left[s \geq r_{n}\right] \leq E\left[\theta^{\prime}(s)\right] \\
& \leq E\left[\frac{w_{U}-w_{n}-\left(s-r_{n}\right) m_{n}}{r_{U}-s} \mid s \geq r_{n}\right] \operatorname{Pr}\left[s \geq r_{n}\right]+E\left[m_{n} \mid s<r_{n}\right] \operatorname{Pr}\left[s<r_{n}\right] \equiv U B_{8(s)}
\end{aligned}
$$

Proof. Illustrated in Figure 5. Logic as in earlier propositions. To maximize $\theta_{n}^{\prime}(s)$, set $\theta_{n}(s)$ at its Proposition 7 lower bound and search over $t>s$ for the Proposition 7 upper bound $\theta_{n}(t)$ that minimizes $\frac{\theta_{n}(t)-\theta_{n}(s)}{t-s}$. For $s>r_{n}$, the relevant $t=r_{U}$. For $s<r_{n}$, $t=r_{n}$.

\section{Application: the value of a statistical life (VSL)}

\subsection{Data}

The data was originally described and analyzed in Leeth and Ruser (2003a). Individualspecific data is drawn from the Current Population Survey (CPS) over the years 1996 to 
1998, producing a cross-section of 158,756 observations. These data include individual hourly wage $w$ as well as individual and job covariates used in explaining wages $a$, such as indicators of usually works less than 20 hours per week, usually works 45 or more hours per week, union member, married, major industry group, major occupation group, race, gender, born outside US of foreign parents, and educational attainment. Age is also given. Characteristics of individual's home town and state are included in the wage covariate vector to allow for compensating differentials for local conditions. These variables include indicators for hometown population size, residence in a central city, and state of residence as well as a continuous measure of the state's workers compensation generosity. Workers in the public, mining, agricultural and a few other sectors are omitted. To avoid problems with top-coded wages, individuals with wages of $\$ 99$ or more were dropped. The CPS data also includes each individual's 3-digit census occupational code.

One hopes to include all compensated differences between jobs in the model. Otherwise, estimates of the price of marginal differences in risk could be biased. To facilitate this kind of analysis, the Bureau of Labor Statistics (BLS) developed "generic leveling factors" measuring 10 dimensions of jobs' duties and responsibilities and designed to capture differences between jobs. As part of the National Compensation Survey, jobs were rated along these dimensions. Leeth and Ruser generated a score for each 3-digit occupation by calculating an average in each dimension for the workers in that occupation. The "work environment" dimension, which is closely correlated with injury risk measures, is omitted. The other 9 leveling variables are included among the wage-influencing convariates $a$.

The fatal and nonfatal injury risk level assigned is the average within the individual's 3 -digit census occupation code. The data contain 434 unique occupations, each with its own fatal and nonfatal injury risk. This risk level reflects the number of injuries over the number of workers in that ocucpation. The number of nonfatal workplace injuries comes from the BLS's Survey of Occupational Injuries and Illnesses. The number of fatal injuries comes from the Census of Fatal Occupational Injuries. These are the numerators in the risk levels. The number of full-time equivalent employees in each occupation is from the CPS. Leeth and Ruser (2003b) describe the procedure used to construct these risk measures in more detail. ${ }^{8}$ The fatal injury risk is $r$. Nonfatal injury risk is considered another job attribute and included in $a$. The correlation between fatal and nonfatal risk is 0.43 , leaving some independent variation in the two types of risk.

Table 1 presents summary statistics on selected variables' sample distribution. The empirical distribution of fatal risk is presented in Figure 6. Consequently, 82 percent of observations have fatal risk levels below the mean. For this reason, this paper focuses on VSL estimates at the the median level of fatal risk. Also, in some analysis, observations at high levels of risk are trimmed.

\footnotetext{
${ }^{8}$ There are two differences between the data they and I use. They analyze men and women separately to investigate gender differences in the risk premium; I pool men and women together. Second, they use race and gender specific risk occupational risk measures; I use the risk measures unconditioned on race and gender.
} 


\section{Analysis}

\subsection{Estimating the market price function $P$}

The most common empirical approach assumes some form of parametric $P$. In their review of the VSL literature, Viscusi and Aldy (2003) mention only that quadratic risk terms are sometimes significant and make no mention of semiparametric or nonparametric methods. Heckman, Matzkin, and Nesheim (2003) use simulation to show that hedonic models generally yield highly nonlinear market price curves. Therefore, parametric methods may obscure important features of the price function. However, at this stage, to keep attention focused on the innovation at hand, a parametric model is assumed. In this vein, we follow Leeth and Ruser (2003a) and assume that $P$ is quadratic in fatal risk. This also allows a simple test of $P$ concave (C5): is $\beta_{2} \leq 0$ ?

$$
\log (w)=\beta_{0}+\beta_{1} r+\beta_{2} r^{2}+\alpha a+v
$$

To assess sensitivity, estimates from 3 other specifications are presented. Specifications vary by the order of fatal and nonfatal risk terms included. For brevity, the specifications will be referred to by the pair order(fatal risk) - order(nonfatal risk). For instance, specification 2-1 has linear and squared fatal risk terms and a linear nonfatal risk term. We analyze 2-0, 0-1, 2-1, and 2-2. All equations also include the other worker and job characteristics described above in $a .^{9}$

Table 2 presents the regression estimates. It is common to interpret the slope of the price function with respect to risk as AWTP and, hence VSL, which can be justified given homogeneous preferences. The table's bottom panel describes the VSL estimates implied by these four specifications. Evaluated at median fatal risk level, the VSL estimates range from $\$ 3.0$ million when nonfatal risk terms are included to $\$ 6.3$ million when they are omitted. Evaluating at the mean fatal risk level, rather than the median, changes each estimate by very little. These results are consistent with the conventional VSL literature, which typically uses these same methods and obtains VSL estimates in the $\$ 4$ - 9 million range [Viscusi and Aldy (2003)].

Two standard errors for each estimated coefficient are presented. Standard errors robust to heteroskedasticity appear in parentheses. Those corrected for correlation of errors within occupation appear in brackets. Assigning the same fatal and nonfatal injury risk levels to all individuals within the same 3-digit occupation undoubtedly produces withinoccupation correlation of errors making the bracketed ones more appropriate. To reflect that uncertainty in the VSL estimate, Table 2 also includes standard errors for the VSL estimates correcting for within-occupation correlation of errors.

Even under the strong homogeneous preference assumptions required here, the VSL estimates are somewhat imprecise. The $95 \%$ confidence intervals for the VSL estimates at

\footnotetext{
${ }^{9}$ Regressions also include as independent variables a constant, age, age squared, nine occupation generic leveling variables, a measure of state workers' compensation generosity, and binary variables for four race categories, nine levels of completed education, married, foreign born, usually works less than 20 hours per week, usually works 45 or more hours per week, union member, eleven major occupations, six major industries, residence in a central city, six city population sizes, and residence in each of 50 states or D.C.
} 
mean risk level range from $-\$ 0.6$ to 11.7 million under $2-0$, from $-\$ 3.2$ to 9.0 million under 2-1 and 2-2. The results are similar when the VSL is estimated at the median level of risk. These are wide ranges. The range of VSL values they cover would have widely different policy implications if interpreted naively.

\subsection{Partial identification of AWTP/VSL}

We present bounds on the VSL estimated both under the shape restrictions alone (C1-2) and assuming smooth, concave $P$ (C1-5), applying the results of Propositions 3 and 7 respectively. Results are presented in Table 3 and described here. Without using information about the price function and using the least conservative parameters $\left(r_{U}=2.1, w_{U}=\$ 107\right)$, the VSL is estimated to be bounded between $\$ 0$ and $U \hat{B}_{3}=\$ 75$ million. With equilibrium and a smooth, concave price function, both the upper and lower bounds tighten to put VSL between $L \hat{B}_{8}=\$ 2.3$ and $U \hat{B}_{8}=\$ 58$ million. These bounds are wide enough to include the majority of estimates in the literature, although they do exclude the estimates found by Ashenfelter and Greenstone (2004) and Powell (2009).

The bounds are sensitive to the choice of values for $r_{U}$ and $w_{U}$, as described theoretically at the end of section 2.2 . This is explored with reference to $U \hat{B}_{3}$, although the same patterns hold for $L \hat{B}_{8}$ and $U \hat{B}_{8}$. The choice of parameters can be informed by the distribution of the data as well as the logical bounds. We can restrict attention to a subset of the logical bounds that holds but does not greatly exceed most of the values present in our data. This allows one to focus on the area where data is dense and not try to analyze sparse-data areas. In analyzing fatal risk, logically $r_{L}=0$ and $r_{U}=1$. However, on this scale, all the fatal occupational risk data is in the $[0,0.0025)$ interval, i.e. from 0 to 250 fatalities per 100,000 FTE workers per year. More than 95 percent of the data is in less than a tenth of that interval: [0, 0.0002) or from 0 to 20 fatalities per 100,000 FTE. Setting the bound on the wage space is more difficult. There is no logical upper bounds on the level of wages a worker might need to remain indifferent between her observed job and an otherwise similar job at a different risk level. Ultimately, the choice is somewhat ad hoc.

In order to illustrate the sensitivity of the estimates to the $\left(r_{U}, w_{U}\right)$ parameters, data are held constant and estimation is carried out across various combinations of $\left(r_{U}, w_{U}\right)$. Two graphs with estimates of the functions $U \hat{B}_{3}$ are presented: figures 7 and 8 . They differ primarily in the value of $r_{U}$ used and secondarily in the range of $w_{U}$ values chosen. Consequently, they also differ in scale. Figure 7 uses $r_{U}=250$, a value 10 percent above the maximum risk level observed in the data. Figure 8 uses $r_{U}=2.1$, a value just above the median. With $r_{U}=2.1$, not all the data can be included in the analysis. In order to hold data constant across the two analyses, observations with risk levels above $r>2$ are trimmed from both. They constitute 35 percent of the data.

Each figure presents four estimated bounds, distinguished by the choice of $w_{U}$. These values were chosen as multiples of the maximum wage observed in the data. A range of values is chosen to illustrate how estimated bounds respond to this parameter. Ideally, the choice of $w_{U}$ should satisfy $w_{U}>\max _{n \in N} \theta_{n}\left(r_{U}\right)$. That is, it should exceed the highest wage needed to keep each individual indifferent between their observed job and a job with risk $r_{U}$. However, there is no obvious way to implement this. 
Consider the results in Figure 7. With the larger $r_{U}$ and at the median risk level $(\operatorname{Median}(r)=1.4)$, the upper bound on the VSL ranges from $\$ 75$ million under the assumption that $w_{U}=\$ 107$ to about $\$ 465$ million under the assumption that $w_{U}=\$ 491$. As the risk level analyzed increases, so does the bound.

Assuming smaller $r_{U}$ increases the bounds for all values of $w_{U}$ and all risk levels. Figure 8 illustrates how dramatically the bounds can change. Now, still using the median risk level and the same values of $w_{U}$, the VSL bound ranges from $\$ 28$ billion to $\$ 173$ billion (not million). Reducing $r_{U}$ by a factor of 120 increased the bound on the VSL by a factor of 2,700 for $w_{U}=\$ 107$.

To further illustrate how the estimated bound on the VSL responds to the choice of parameters $r_{U}$ and $w_{U}$, consider Figure 9 . It presents the estimated upper bound on the VSL evaluated at the median fatal risk level $-U \hat{B}_{3}(1.4)$ - as a function of the two space bounding parameters. These parameters vary across the ranges $r_{U} \in[2.1,290]$ and $w_{U} \in[107,584]$. As expected from the comparative statics, the tightest bound is attained when $r_{U}$ is highest and $w_{U}$ is lowest. It rises dramatically as $r_{U}$ approaches the region containing the data. As stated before, this bound could be made arbitrarily large by setting $w_{U}$ arbitrarily large or $r_{U}$ arbitrarily close to the risk level of interest.

\section{Conclusion}

This paper has implications for both policymakers and economists. For policymakers, it warns that the VSL estimates produced by common types of analysis are sensitive to the particular, strong assumptions made. In fact, under other assumptions, the data are consistent with a wide range of VSL estimates. This should give policy makers pause when looking to these estimates to guide life-and-death decisions.

For economists, the paper introduces a new approach for hedonic analysis of crosssectional data in search of conclusions about WTP. Under a weak set of assumptions on preferences, we have developed a new way to obtain informative bounds on WTP under a range of conditions on market structure. However, the bounds are wide and sensitive to parameter choice. This is an accurate reflection of the inescapable, underlying fact that a single observation on each individual does not give you much information to identify features of their preferences. Of course, one can always assume enough structure to say more but the conclusions are only as credible as the assumptions.

Although this paper develops the framework in the context of VSL, its approach will translate directly into other areas of economics that use hedonic analysis in order to study willingness to pay for changes in the hedonic attribute. Rather than only presenting point estimates based on strong structural assumptions, analysts can use this approach to also explore what the data imply under other, weaker conditions. 


\section{References}

Ashenfelter, O., and M. Greenstone (2004): "Using Mandated Speed Limits to Measure the Value of a Statistical Life," Journal of Political Economy, 112(1).

Bajari, P., and C. L. Benkard (2005): "Demand Estimation with Heterogeneous Consumers and Unobserved Product Characteristics: A Hedonic Approach," Journal of Political Economy, 113(6).

Biddle, J. E., And G. A. Zarkin (1988): "Worker Preference and Market Compensation for Job Risk," Review of Economics and Statistics, 70(4).

Black, S. E. (1999): "Do Better Schools Matter? Parental Valuation of Elementary Education," Quarterly Journal of Economics, 114(2), 577-599.

Brown, C. (1980): "Equalizing Differences in the Labor Market," Quarterly Journal of Economics, 94(1), 113-134.

Chay, K., and M. Greenstone (2005): "Does Air Quality Matter? Evidence from the Housing Market," Journal of Political Economy, 113(2).

Duval, T., And D. J. Gribbin (2009): "Memo to Secretarial Officers and Modal Administrators regarding Treatment of the Economic Value of a Statistical Life in Departmental Analyses," Discussion paper, U.S. Department of Transportation.

Eckland, I., J. Heckman, and L. Nesheim (2004): "Identification and Estimation of Hedonic Models," Journal of Political Economy, 112(1).

Heckman, J., R. Matzkin, and L. Nesheim (2003): "Simulation and Estimation of Hedonic Models," .

Heckman, J. J., R. L. Matzkin, and L. Nesheim (2009): "Nonparametric Identification and Estimation of Nonadditive Hedonic Models," IZA Discussion Paper Number 4329 .

Hwang, H.-S., W. R. Reed, and C. Hubbard (1992): "Compensating Wage Differentials and Unobserved Productivity," Journal of Political Economy, 100(4), 835-858.

Kochi, I., B. Hubbell, and R. Kramer (2006): "An empirical Bayes approach to combining and comparing estimates of the value of a statistical life for environmental policy analysis," Environmental and Resource Economics, 34(3), 385-406.

Konüs, A. A. (1939): "The Problem of the True Index of the Cost of Living, translated in," Econometrica, 7, 10-29.

Leeth, J. D., And J. Ruser (2003a): "Compensating Wage Differentials for Fatal and Nonfatal Injury Risk by Gender and Race," The Journal of Risk and Uncertainty, 27(3), $257-277$. 
(2003b): "Occupational Injury Rates by Race and Gender," Department of Economics, Bentley College, photocopy.

Manski, C. F. (2003): Partial Identification of Probability Distributions. Springer-Verlag.

Miller, T. (2000): "Variations between countries in values of statistical life," Journal of Transport Economics and Policy, pp. 169-188.

MrozeK, J., And L. TAYlor (2002): "What determines the value of life? A metaanalysis," Journal of Policy Analysis and Management, pp. 253-270.

OF LABOR Statistics, B. (2005): "Number and rate of fatal occupational injuries by major occupation groups," Bureau of Labor Statistics.

PAKes, A. (2003): "A Reconsideration of Hedonic Prices Indices with an Application to PC's," American Economic Review, 93.

PAkes, A. (2008): "Hedonics and the Consumer Price Index," forthcoming in," Les Annales d'Economie et de Statistique.

Powell, D. (2009): "Using Income Tax Changes to Identify the Value of a Statistical Life," Working paper.

Pratt, J. W., and R. J. Zeckhauser (1996): "Willingness to Pay and the Distribution of Risk and Wealth," Journal of Political Economy, 104(4), 747-763.

Preston, S. H., And E. Buzzell (2006): "Service in Iraq: Just How Risky?," Washington Post.

Rosen, S. (1986): The Theory of Equalizing Differencesvol. I, chap. 12, pp. 641-692. Elsevier Scientific Publishers.

Rosen, S., And R. Thaler (1976): "The value of saving a life: Evidence from the labor market," NBER Working Paper.

Shogren, J. F., And T. Stamland (2002): "Skill and the Value of Life," Journal of Political Economy, 110(5), 1168-1173.

Smith, V., And J. HuAng (1995): "Can markets value air quality? A meta-analysis of hedonic property value models," The Journal of Political Economy, 103(1), 209-227.

Viscusi, W. K. (1992): Fatal Tradeoffs: Public and Private Responsibilities for Risk. Oxford University Press, New York.

(2004): "The Value of Life: Estimates with Risks by Occupation and Industry," Economic Inquiry, 42(1), 169-188.

Viscusi, W. K., And J. Aldy (2003): "The Value of a Statistical Life: A Critical Review of Market Estimates Throughout the World," The Journal of Risk and Uncertainty, $27(1), 5-76$. 
Viscusi, W. K., And W. N. Evans (1990): "Utility Functions That Depend on Health Status: Estimates and Economic Implications," The American Economic Review, 80(3), 353-374. 


\section{Appendix: Proofs and supporting results}

This appendix begins by defining conditions and proving lemmas, which are useful in proving the propositions in the main text. These results build on Manski (2003) section

8.4. I adapt those to the increasing, convex case and integrate them with equilibrium conditions.

\subsection{Supporting results}

Consider two functions $P_{n}$ and $\theta_{n}$ defined from the domain $\left[r_{L}, r_{U}\right]$ to the range $\left[w_{L}, w_{U}\right]$. Any of these boundaries may be infinite unless noted. We are studying any individual- $n$ 's status-quo indifference curve as $r$ varies but holding all other variables fixed, $\theta_{n}$. When we consider the relationship between individual-n's indifference curve and the price function $P$, we express $P$ only as a function only of $r$, conditioning on the status-quo values for all other variables and suppressing them. It is denoted $P_{n} \equiv P\left(r, a=a_{n}, x=x_{n}, v=v_{n}\right)$

Define the following conditions $\forall r, s, t \in\left[r_{L}, r_{U}\right]$ with $r<s<t$. Condition 6 captures the fact that the indifference curve is defined relative to the observed status-quo job. Condition 7 expresses the fact that, because the status-quo job must be in the individual's choice set, it must belong to the price function or the market-opportunity curve. All these conditions would apply even if the wage (price) function were discrete and discontinuous.

Condition 6 (One point on $\theta_{n}$ known). : $\exists r_{n} \in\left[r_{L}, r_{U}\right]$ such that $w_{n} \equiv \theta_{n}\left(r_{n}\right)$ known.

Condition 7 (Equal at known point). : $\exists r_{n} \in\left[r_{L}, r_{U}\right]$ s.t. $\theta_{n}\left(r_{n}\right)=P_{n}\left(r_{n}\right)$ known.

Lemma 9 exploits convexity to put an upper bound on the indifference curve at risk levels higher than $r_{n}$. Intuitively, if the indifference curve were to take values above this bound, then it would have to attain a value higher than $w_{U}$ at $r_{U}$, which would violate the bounded risk-wage space. Lemma 10 uses similar logic to put a lower bound on the indifference curve for risk levels below $r_{n}$. These are used for proving Proposition 1.

Lemma 9 (Upper bound on convex $\theta_{n}$ at higher values). Given $C 1 . b$ and $C 6$, then $\forall s, t$ with $r_{n}<s<t$,

$$
\theta_{n}(s) \leq \frac{w_{U}-w_{n}}{t-r_{n}}\left(s-r_{n}\right)+w_{n}
$$

Proof.

$$
\begin{aligned}
w_{U} \geq \theta_{n}(t) & =\frac{\theta_{n}(t)-\theta_{n}(s)}{t-s}(t-s)+\theta_{n}(s) \\
& =\frac{\theta_{n}(t)-\theta_{n}(s)}{t-s}(t-s)+\frac{\theta_{n}(s)-\theta_{n}\left(r_{n}\right)}{s-r_{n}}\left(s-r_{n}\right)+\theta_{n}\left(r_{n}\right) \\
& \geq \frac{\theta_{n}(s)-\theta_{n}\left(r_{n}\right)}{s-r_{n}}(t-s)+\frac{\theta_{n}(s)-\theta_{n}\left(r_{n}\right)}{s-r_{n}}\left(s-r_{n}\right)+\theta_{n}\left(r_{n}\right) \\
& =\frac{\theta_{n}(s)-\theta_{n}\left(r_{n}\right)}{s-r_{n}}\left(t-r_{n}\right)+\theta_{n}\left(r_{n}\right)
\end{aligned}
$$


The first line comes by definition of $w_{U}$ and by expressing $\theta_{n}(t)$ as a linear function connected to $\left(s, \theta_{n}(s)\right)$. The second comes by similarly expressing $\theta_{n}(s)$ with reference to $\left(r, \theta_{n}(r)\right)$. $C 1 . b$ gives the third. The fourth collects terms. Rearranging to solve for $\theta_{n}(s)$ produces the stated bound.

Lemma 10 (Backcasting). Given C1.b, for $a<b<c$,

$$
\theta_{n}(a) \geq \theta_{n}(b)+\frac{\theta_{n}(c)-\theta_{n}(b)}{c-b}(a-b)
$$

Proof. By C1.b, $\frac{\theta_{n}(b)-\theta_{n}(a)}{b-a} \leq \frac{\theta_{n}(c)-\theta_{n}(b)}{c-b}$. Rearranging yields the lower bound.

$$
\begin{aligned}
\theta_{n}(b)-\theta_{n}(a) & \leq \frac{\theta_{n}(c)-\theta_{n}(b)}{c-b}(b-a) \\
\theta_{n}(b)+\frac{\theta_{n}(c)-\theta_{n}(b)}{c-b}(a-b) & \leq \theta_{n}(a)
\end{aligned}
$$

Lemma 11 (Lower bound on $\theta$ at values higher than $r_{n}$ ). Given $C 1 . b \cap C 3 \cap C 6 \cap C 7$, then

$$
\forall t>r_{n}, \quad \theta_{n}(t) \geq \theta_{n}\left(r_{n}\right)+\max _{s \in\left(r_{n}, t\right)} \frac{P_{n}(s)-P_{n}\left(r_{n}\right)}{s-r_{n}}\left(t-r_{n}\right)
$$

Proof. For $\forall s \in\left(r_{n}, t\right), \frac{P_{n}(s)-P_{n}\left(r_{n}\right)}{s-r_{n}} \leq \frac{\theta_{n}(s)-\theta_{n}\left(r_{n}\right)}{s-r_{n}} \leq \frac{\theta_{n}(t)-\theta_{n}(s)}{t-s}$. The first inequality comes from $C 3$ and $C 7$, the second from $C 1 . b$.

We can express our object of interest in point-slope form for the line passing between $\left(s, \theta_{n}(s)\right)$ and $\left(t, \theta_{n}(t)\right)$ as

$$
\theta_{n}(t)=\theta_{n}(s)+\frac{\theta_{n}(t)-\theta_{n}(s)}{t-s}(t-s)
$$

By substituting in a similar expression for $\theta_{n}(s)$ and using the two inequalities obtained above and consolidating the last two terms in (9), we obtain a lower bound on $\theta_{n}(t)$,

$$
\begin{aligned}
\theta_{n}(t) & = & \theta_{n}\left(r_{n}\right)+\frac{\theta_{n}(s)-\theta_{n}\left(r_{n}\right)}{s-r_{n}}\left(s-r_{n}\right)+\frac{\theta_{n}(t)-\theta_{n}(s)}{t-s}(t-s) \\
& \geq & \theta_{n}\left(r_{n}\right)+\frac{P_{n}(s)-P_{n}\left(r_{n}\right)}{s-r_{n}}\left(s-r_{n}\right)+\frac{P_{n}(s)-P_{n}\left(r_{n}\right)}{s-r_{n}}(t-s) \\
& = & \theta_{n}\left(r_{n}\right)+\frac{P_{n}(s)-P_{n}\left(r_{n}\right)}{s-r_{n}}\left(t-r_{n}\right)
\end{aligned}
$$

This defines a lower bound for each $s \in\left(r_{n}, t\right)$. We obtain the greatest lower bound on $\theta_{n}(t)$ by considering the maximum across all such $s$.

Lemma 12 (Lower bound on $\theta$ at values lower than $r_{n}$ ). Given $C 1 . b \cap C 3 \cap C 6 \cap C 7$, then

$$
\forall p<r_{n}, \quad \theta_{n}(p) \geq \theta_{n}\left(r_{n}\right)-\min _{q \in\left(p, r_{n}\right)}\left\{\frac{P_{n}\left(r_{n}\right)-P_{n}(q)}{r_{n}-q}\right\}\left(r_{n}-p\right)
$$


Proof. As in the proof of lemma 11. For $\forall q \in\left(p, r_{n}\right), \frac{P_{n}\left(r_{n}\right)-P_{n}(q)}{r_{n}-q} \geq \frac{\theta_{n}\left(r_{n}\right)-\theta_{n}(q)}{r_{n}-q} \geq \frac{\theta_{n}(q)-\theta_{n}(p)}{q-p}$. Express our object of interest in point-slope form for the line passing between $\left(q, \theta_{n}(q)\right)$ and $\left(p, \theta_{n}(p)\right)$ as

$$
\theta_{n}(p)=\theta_{n}(q)-\frac{\theta_{n}(q)-\theta_{n}(p)}{q-p}(q-p)
$$

As above, we obtain a lower bound on $\theta_{n}(p)$,

$$
\begin{aligned}
& \theta_{n}(p)=\theta_{n}\left(r_{n}\right)-\frac{\theta_{n}\left(r_{n}\right)-\theta_{n}(q)}{r_{n}-q}\left(r_{n}-q\right)-\frac{\theta_{n}(q)-\theta_{n}(p)}{q-p}(q-p) \\
& \geq \theta_{n}\left(r_{n}\right)-\frac{\theta_{n}\left(r_{n}\right)-\theta_{n}(q)}{r_{n}-q}\left(r_{n}-q\right)-\frac{\theta_{n}(q)-\theta_{n}(p)}{q-p}(q-p) \\
& =\quad \theta_{n}\left(r_{n}\right)-\frac{\theta_{n}\left(r_{n}\right)-\theta_{n}(q)}{r_{n}-q}\left(r_{n}-p\right)
\end{aligned}
$$

This defines a lower bound for each $q \in\left(p, r_{n}\right)$. We obtain the greatest lower bound on $\theta_{n}(p)$ by considering the minimum across all such $q$. 


\section{$\begin{array}{ll}7 & \text { Tables }\end{array}$}

Table 1: Sample summary statistics

\begin{tabular}{|c|c|c|c|c|c|}
\hline \hline Variable & Mean & S.D. & Med. & Min. & Max. \\
\hline Hourly wage & 14.08 & 8.20 & & 2.21 & 98.13 \\
Fatal injury risk/100,000FTE & 3.71 & 8.27 & 1.40 & 0 & 226.7 \\
Nonfatal injury risk/100FTE & 1.83 & 2.07 & 1.04 & 0 & 16.7 \\
Age & 37.6 & 11.5 & & 18 & 65 \\
Black & & & & & \\
Hispanic & 0.090 & & & & \\
Other non-white & 0.088 & & & & \\
High school grad. only & 0.35 & & & & \\
Some college, no degree & 0.22 & & & & \\
Vocational assoc. degree & 0.05 & & & & \\
Academic assoc. degree & 0.04 & & & & \\
Bachelors degree & 0.17 & & & & \\
Masters degree & 0.04 & & & & \\
Professional degree & 0.01 & & & & \\
PhD & 0.01 & & & & \\
Less than 20 hours/week & 0.04 & & & & \\
More than 40 hours/week & 0.19 & & & & \\
Foreign-born & 0.11 & & & & \\
Union member & 0.10 & & & & \\
City & 0.23 & & & & \\
\hline
\end{tabular}


Table 2: Results from various parametric specifications of model 1

\begin{tabular}{ccccc}
\hline \hline Dependent variable: $\log ($ wage $)$ & \multicolumn{4}{c}{ Specification } \\
Independent variables & $2-0$ & $0-1$ & $2-1$ & $2-2$ \\
\hline \hline Fatal injury risk/100 & 0.251 & & 0.119 & 0.123 \\
& $(0.031)^{* *}$ & & $(0.031)^{* *}$ & $(0.031)^{* *}$ \\
& {$[0.116]^{*}$} & & {$[0.127]$} & {$[0.125]$} \\
& $($ Fatal injury risk/100) & & \\
& -0.136 & & -0.060 & -0.052 \\
& $(0.027)^{* *}$ & & $(0.027)^{*}$ & $(0.027)$ \\
& {$[0.067]^{*}$} & & {$[0.071]$} & {$[0.072]$} \\
Nonfatal injury risk/100 & & 1.766 & 1.687 & 4.505 \\
& & $(0.085)^{* *}$ & $(0.087)^{* *}$ & $(0.203)^{* *}$ \\
& & {$[0.586]^{* *}$} & {$[0.599]^{* *}$} & {$[1.598]^{* *}$} \\
$($ Nonfatal injury risk/100) & & & & -28.10 \\
& & & & $(1.88)^{* *}$ \\
& & & & {$[11.606]^{*}$} \\
$\widehat{V S L}_{H P}($ Mean $(r))$, millions & $\$ 6.2$ & & $\$ 2.9$ & $\$ 3.1$ \\
& {$[2.8]$} & & {$[3.10]$} & {$[3.06]$} \\
$\widehat{V S L}_{H P}($ Median $(r))$, millions & $\$ 6.3$ & & $\$ 3.0$ & $\$ 3.1$ \\
& {$[2.9]$} & & {$[3.18]$} & {$[3.14]$} \\
\hline
\end{tabular}

* (**) denotes significance at the 5 (1) percent level.

Table 3: Estimated bounds on VSL assuming shape restrictions on indifference curves $C 1-2,\left(r_{U}, w_{U}\right)=(2.1, \$ 107)$, and trimming data with $r_{n}>2$

\begin{tabular}{|c|cc|}
\hline \hline Conditions on price function & Lower bound & Upper bound \\
\hline & $L \hat{B}_{3}$ & $U \hat{B}_{3}$ \\
& 0 & $\$ 75$ million \\
& & \\
& $L \hat{B}_{8}$ & $U \hat{B}_{8}$ \\
Sme & $\$ 2.3$ million & $\$ 58$ million \\
\hline
\end{tabular}




\section{Figures}

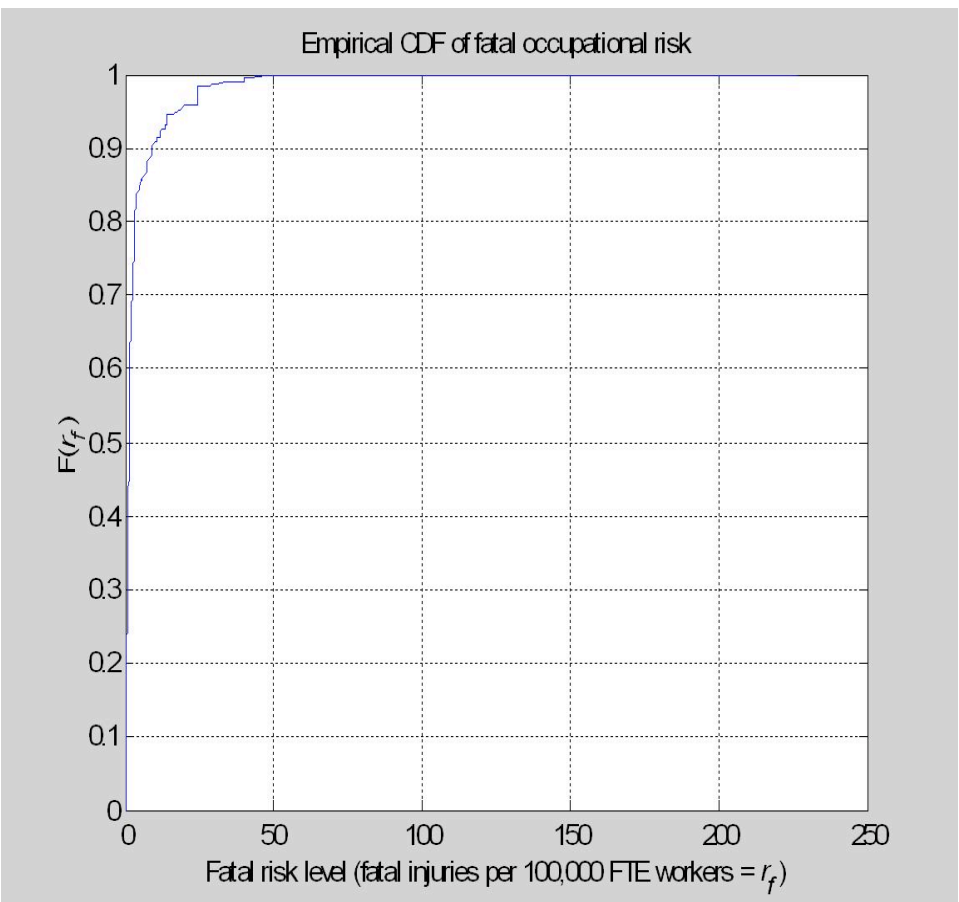

Figure 6: Distribution of fatal occupational risk in the sample. The data contain a few people in very high risk occupations. Eighty-two percent are at levels below the mean level. 


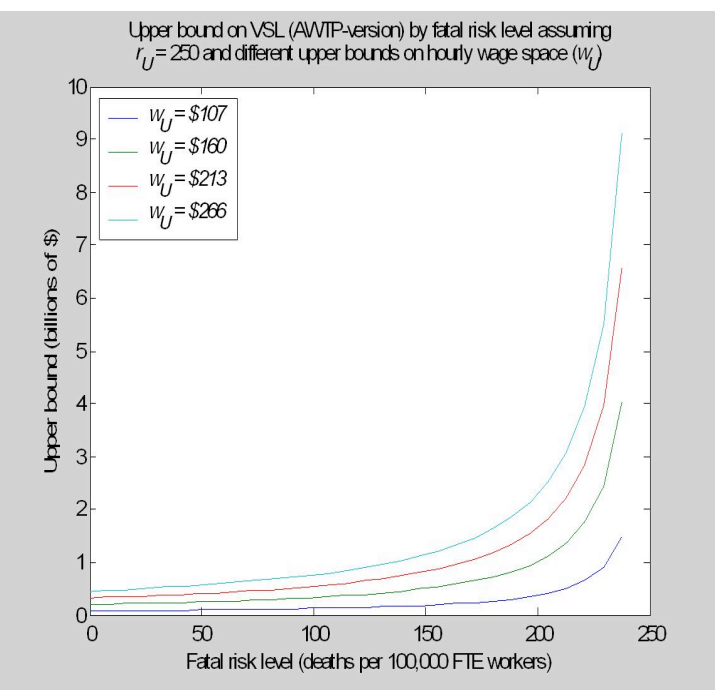

Figure 7: $U \hat{B}$ assuming $r_{U}=250$ and various $w_{U}$.

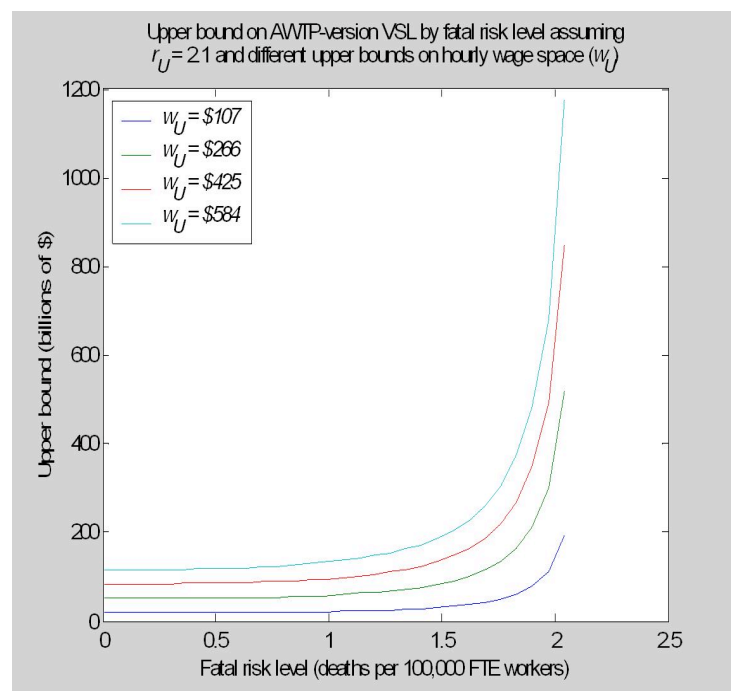

Figure 8: $U \hat{B}$ assuming $r_{U}=2.1$ and various $w_{U}$. 


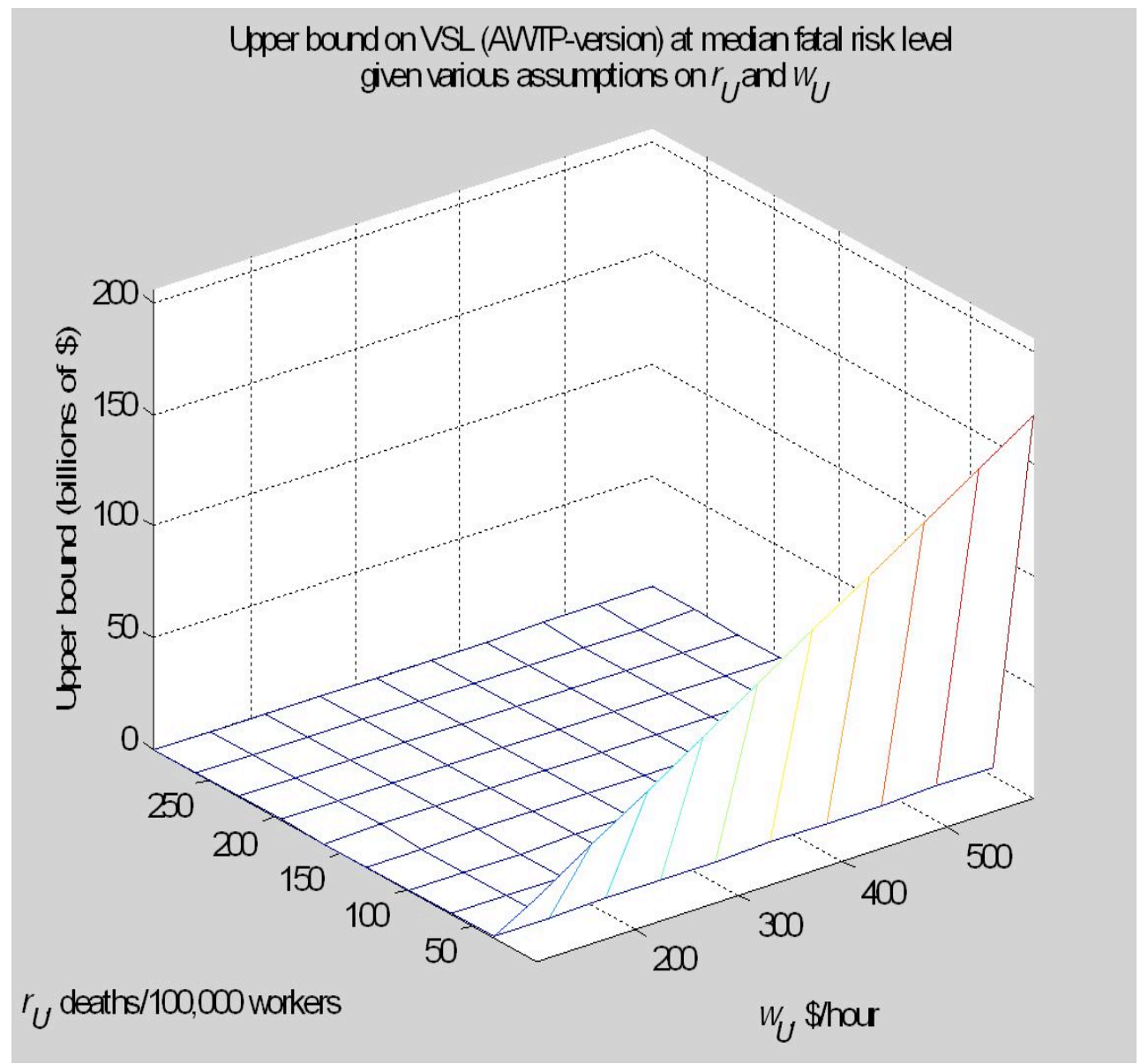

Figure 9: Upper bound on VSL at median $(r)$ under various parameter values. 